\title{
NLL+NNLO predictions for jet-veto efficiencies in Higgs-boson and Drell-Yan production
}

\author{
Andrea Banfi, ${ }^{a}$ Gavin P. Salam ${ }^{b, c, d}$ and Giulia Zanderighi ${ }^{e}$ \\ ${ }^{a}$ Physikalisches Institut, Albert-Ludwigs-Universität Freiburg, \\ D-79104 Freiburg, Germany \\ ${ }^{b} \mathrm{CERN}, \mathrm{PH}-\mathrm{TH}$, \\ CH-1211 Geneva 23, Switzerland \\ ${ }^{c}$ Department of Physics, Princeton University, \\ Princeton, NJ 08544, U.S.A. \\ ${ }^{d}$ LPTHE, CNRS UMR 7589, UPMC Univ. Paris 6, \\ Paris, France \\ ${ }^{e}$ Rudolf Peierls Centre for Theoretical Physics, University of Oxford, \\ 1 Keble Road, Oxford, U.K. \\ E-mail: andrea.banfi@physik.uni-freiburg.de, gavin.salam@cern.ch, \\ g.zanderighi1@physics.ox.ac.uk
}

ABSTRACT: Using the technology of the CAESAR approach to resummation, we examine the jet-veto efficiency in Higgs-boson and Drell-Yan production at hadron colliders and show that at next-to-leading logarithmic (NLL) accuracy the resummation reduces to just a Sudakov form factor. Matching with NNLO calculations results in stable predictions for the case of Drell-Yan production, but reveals substantial uncertainties in gluon-fusion Higgs production, connected in part with the poor behaviour of the perturbative series for the total cross section. We compare our results to those from POWHEG with and without reweighting by HqT, as used experimentally, and observe acceptable agreement. In an appendix we derive the part of the NNLL resummation corrections associated with the radius dependence of the jet algorithm.

KEYwords: Higgs Physics, Resummation, QCD, Standard Model

ArXiv EPRINT: 1203.5773 


\section{Contents}

1 Introduction $\quad 1$

2 The jet-veto efficiency 3

3 NLL resummation $\quad 4$

4 Jet-veto at fixed order $\quad 8$

$\begin{array}{llr}4.1 & \text { Prescriptions for the efficiency } & 9\end{array}$

$\begin{array}{lll}4.2 & \text { Numerical results } & 10\end{array}$

5 Prescription for estimating total uncertainties 12

6 Matching NLL and NNLO results 12

$\begin{array}{lll}6.1 & \text { Matching prescriptions } & 12\end{array}$

$\begin{array}{ll}6.2 \text { Matched results } & 14\end{array}$

$\begin{array}{lll}7 & \text { Comparisons to other calculations } & 17\end{array}$

$\begin{array}{lll}7.1 & \text { Effects beyond the scope of matched calculations } & 17\end{array}$

$\begin{array}{ll}7.2 \text { Comparisons with POWHEG and HqT } & 18\end{array}$

8 Conclusions 21

A NNLL $R$-dependent terms

A.1 Correlated emission 23

$\begin{array}{ll}\text { A.2 Independent emission } & 26\end{array}$

\section{Introduction}

One of the most active topics currently in particle physics is the search for the Higgs boson [1-3]. This scalar boson is the one particle of the standard model that remains to be found, and its discovery would provide the most direct evidence to date that the existence of a Higgs field [4-8] is responsible for electroweak symmetry breaking.

Several production and decay channels are used to search for the Higgs boson at the LHC and Tevatron, with varying sensitivities across the range of Higgs-boson masses allowed in the standard model. One channel with sensitivity over a broad range of masses is gluon-fusion production followed by decay to a $W^{+} W^{-}$pair, one of which may be offshell. For a Higgs mass of about $125 \mathrm{GeV}$, as hinted at by current data [1, 2], this channel is one of several that is expected to be clearly observable with forthcoming data. An accurate 
understanding of its cross section will therefore be crucial in constraining the Higgs-boson couplings in the event of a discovery.

Extensive work has been performed over the past decade to precisely predict the cross sections and branching ratios for all the main production and decay channels, as reviewed for example in $[9,10]$. In the case of Higgs boson production via gluon fusion with subsequent decay to two $W$ bosons, a feature that is of particular importance in discussing cross-section determinations is that it is customary for the LHC experiments to separately treat events according to their different jet multiplicities $[11,12]$. This is because events with no jets are relatively free of backgrounds other than $W^{+} W^{-}$production, while events with one jet have additional backgrounds from $t \bar{t}$ and Drell-Yan production, and events with two jets or more also receive enhanced signal contributions from vector-boson fusion production.

Of particular usefulness for the problem of evaluating cross sections in gluon-fusion production with different jet multiplicities, are fixed-order calculations that allow one to place arbitrary cuts on the final state, up to next-to-next-to-leading order (NNLO) for inclusive production [13-15] and NLO for the production in association with one [16-18] or two jets $[19,20]$. However, the transverse momentum $\left(p_{t}\right)$ thresholds used for identifying jets are usually well below the Higgs mass $\left(M_{H}\right)$. This results in the appearance of logarithmically enhanced terms at all orders of perturbation theory $\alpha_{s}^{n} \ln ^{2 n} M_{H} / p_{t}$, which can spoil the convergence of fixed-order truncations of the series. Such problems arise also when examining other related final-state observables, such as the Higgs-boson or $Z$-boson transverse momentum, and the "beam thrust" [21], for which resummations have been performed to next-to-next-to-leading-logarithmic accuracy (NNLL) and combined with NNLO [22-25], or the transverse energy flow, resummed to next-to-leading-logarithmic [26] accuracy.

Perhaps surprisingly, however, to date no resummation has been performed for Higgsrelated final-state definitions that involve jet finding. This may be because jet related observables are less inclusive than those resummed at high accuracy so far, and therefore require a more detailed understanding of how multiple emissions affect the value of the observable. For example in notable cases, e.g. the $y_{23}$ jet resolution parameter of the exclusive $k_{t}$-algorithm in $e^{+} e^{-}$or hadronic collisions [27, 28], the existing NLL answer has yet to be expressed in anything other than a numerical form [29, 30]. Therefore, instead, the matched NNLL+NNLO results for other observables like the Higgs $p_{t}$ have been used to reweight NLO hadronic event generators, MC@NLO [31] and POWHEG [32] (those generators have been also used in standalone form). Insofar as a jet veto differs from a Higgs- $p_{t}$ veto starting only from order $\alpha_{s}^{2}$ relative to the Born process, the reweighting procedure should provide reasonable predictions. On the other hand, the modelling of inclusive Higgs production processes in MC@NLO or POWHEG is such that the $\alpha_{s}^{2}$ difference between the jet veto and Higgs $p_{t}$ veto is not correctly included. Consequently some of the accuracy of the NNLL+NNLO calculation is lost in the reweighting procedure.

The purpose of this paper is to examine the resummation, matching to fixed order and resulting phenomenology directly for the jet veto observable itself. Because a jet definition is a less inclusive observable than the Higgs-boson $p_{t}$ or the beam thrust, the resummation cannot be immediately reduced to standard forms such as [33]. However, at NLL accuracy, 
to which we shall mostly limit ourselves here, one may make use of the computer automated expert semi-analytical resummer (CAESAR) [34] to perform the resummation. The result turns out to be rather simple and straightforward also to understand analytically.

For Higgs production, throughout this article, we will use the large $m_{\text {top }}$ approximation. This does not affect the resummation at our accuracy but is relevant when we combine the resummation with fixed order calculations.

In parallel with the Higgs-boson case, we will also examine the jet-veto resummation, matching and phenomenology for Drell-Yan production, which has been argued to provide a control case, even if, as we shall see, the issues that arise in the Higgs-boson case are not directly mirrored in Drell-Yan production.

\section{The jet-veto efficiency}

We consider the production of a Higgs or a $\mathrm{Z}$ boson accompanied by $N$ extra QCD partons $p_{1}, \ldots p_{N}$,

$$
p p \rightarrow H+p_{1}+\ldots p_{N}, \quad \text { and } \quad p p \rightarrow Z+p_{1}+\ldots p_{N} .
$$

A jet-veto condition is imposed by clustering the events into jets using a suitable hadroncollider jet-definition (JD) and requiring that the event has no jets with transverse momentum above a certain threshold, typically in the range of $25-30 \mathrm{GeV}$. To define the jets, the LHC experiments usually use the anti- $k_{t}$ algorithm [35], which repeatedly merges the pair of particles with smallest distance measure $d_{i j}=\min \left(p_{t i}^{-2}, p_{t j}^{-2}\right) \Delta R_{i j}^{2} / R^{2}$, unless there exists a particle with a $d_{i B}=p_{t i}^{-2}$ value that is smaller, in which case $i$ becomes a jet and is removed from the list of particles. Here $\Delta R_{i j}^{2}=\left(y_{i}-y_{j}\right)^{2}+\left(\phi_{i}-\phi_{j}\right)^{2}$, where $y_{i}$ and $\phi_{i}$ are respectively the rapidity and azimuth of particle $i$. The parameter $R$ sets the typical angular reach of the jet definition and is often referred to as the jet radius. After the clustering, one may choose to consider all jets, or alternatively only those within some limited rapidity range, in reflection of actual experimental acceptances.

The cross section with a jet-veto is defined as

$$
\Sigma\left(p_{\mathrm{t}, \mathrm{veto}}\right)=\sum_{N} \int d \Phi_{N} \frac{d \sigma_{N}}{d \Phi_{N}} \Theta\left(p_{\mathrm{t}, \mathrm{veto}}-p_{\mathrm{t}, \mathrm{j} 1}^{(\mathrm{JD})}\left(p_{1}, \ldots, p_{N}\right)\right)
$$

where $p_{\mathrm{t}, \mathrm{j} 1}$ is the transverse momentum of the hardest (highest $p_{t}$ ) of the jets found in the event, $d \sigma_{N}$ denotes the partonic cross-section to produce a Higgs or a $\mathrm{Z}$ boson accompanied by $N$ extra partons and $d \Phi_{N}$ is the corresponding phase space.

It is also useful to consider the jet-veto efficiency defined as

$$
\epsilon\left(p_{\mathrm{t}, \mathrm{veto}}\right) \equiv \frac{\Sigma\left(p_{\mathrm{t}, \mathrm{veto}}\right)}{\sigma},
$$

where $\sigma$ is the total cross-section. The veto efficiency is of interest because it essentially encodes just the information about the Sudakov suppression associated with forbidding radiation of jets. In contrast, the vetoed cross section mixes in also the physics that determines the total cross section. Thus, in the absence of a veto, $p_{\mathrm{t}, \text { veto }}=\infty$, the efficiency is exactly 1 and one can reliably discuss small departures from $\epsilon=1$ as $p_{\text {t,veto }}$ is reduced. In 
the vetoed cross section, $\Sigma\left(p_{\text {t,veto }}\right)$, it is harder to disentangle those effects from uncertainties on the total cross section. Later, we will argue that even for $\epsilon$ substantially below 1, it makes sense to treat the efficiency and total cross section as independent quantities, and that the uncertainties that govern them are relatively uncorrelated.

Each of $\Sigma\left(p_{\mathrm{t}, \text { veto }}\right), \sigma$ and $\epsilon\left(p_{\mathrm{t}, \text { veto }}\right)$ has a fixed-order perturbative expansion, which we write as

$$
\begin{aligned}
\Sigma\left(p_{\mathrm{t}, \text { veto }}\right) & =\Sigma_{0}\left(p_{\mathrm{t}, \text { veto }}\right)+\Sigma_{1}\left(p_{\mathrm{t}, \text { veto }}\right)+\Sigma_{2}\left(p_{\mathrm{t}, \text { veto }}\right)+\ldots, \\
\epsilon\left(p_{\mathrm{t}, \text { veto }}\right) & =\epsilon_{0}\left(p_{\mathrm{t}, \text { veto }}\right)+\epsilon_{1}\left(p_{\mathrm{t}, \text { veto }}\right)+\epsilon_{2}\left(p_{\mathrm{t}, \text { veto }}\right)+\ldots, \\
\sigma & =\sigma_{0}+\sigma_{1}+\sigma_{2}+\ldots,
\end{aligned}
$$

where the index $i$ signifies that the contribution is proportional to $\alpha_{s}^{i}$ relative to the Born term. The properties $\Sigma_{0}\left(p_{\text {t,veto }}\right) \equiv \sigma_{0}$ and $\epsilon_{0}=1$ follow from the fact that no jets are present at Born level.

\section{NLL resummation}

The jet-veto efficiency is nothing but the cumulative distribution of the transverse momentum of the highest- $p_{t}$ jet, $p_{\mathrm{t}, \mathrm{j} 1}$. In order to have a dimensionless observable, we choose to divide it by the corresponding boson mass, $M_{B}=M_{Z}$ or $M_{H}$,

$$
V\left(\tilde{p}_{1}, \tilde{p}_{2} ; k_{1} \ldots k_{N}\right)=\frac{p_{\mathrm{t}, \mathrm{j} 1}\left(k_{1} \ldots k_{N}\right)}{M_{B}} .
$$

Here $\tilde{p}_{1}$ and $\tilde{p}_{2}$ denote the incoming partons entering the hard scattering (after any initial state emission), $k_{1} \ldots k_{N}$ are the momenta of the final-state QCD partons. The momentum of the boson can be obtained using energy-momentum conservation.

We present here a next-to-leading logarithmic (NLL) resummation of the jet-veto efficiency, i.e. we resum all logarithms in $\ln \epsilon\left(p_{\mathrm{t} \text {,veto }}\right)$ up to $\alpha_{s}^{n} L^{n}$, with $L \equiv \ln \left(M_{B} / p_{\mathrm{t}, \text { veto }}\right)$. This observable is within the scope of the computer automated resummation program CAESAR [34]. CAESAR is a program that, given a computer subroutine for an observable, automatically performs a numerical analysis of its behaviour with respect to multiple soft and collinear emissions. From this analysis it establishes whether the observable belongs to a broad class for which it is able to perform NLL resummations, and if so expresses the result for the resummation in terms of a "master formula" together with various numerically determined input parameters. The physics framework that underlies CAESAR can also be used in an analytic context as we shall do, briefly, here.

The first element of the analysis of an observable is to establish its dependence on the kinematics of a single soft and collinear emission. For the case of the jet veto, we simply have

$$
V(\{\tilde{p}\}, k)=\frac{k_{t}}{M_{B}},
$$

where $k_{t}$ is the transverse momentum of the emission with respect to the beam. This expression holds exactly in all (hard, soft, collinear) limits. 
The next element is to establish whether the observable is continuously global [3638]: essentially, the observable should be non-zero for any emission with finite energy and angle with respect to the beam; furthermore, the power-law of the observable's dependence on the emission energy should be independent of the emission angle. Strictly speaking, the first part of this condition holds only if jets are measured at all rapidities, whereas real experimental measurements have finite acceptance. We shall however for now work assuming full acceptance and return to the question in section 7. Given the first part of the condition, the second part holds trivially for the jet veto, and this ensures that the resummation is free of "non-global" logarithms and related terms.

A second condition was dubbed recursive infrared and collinear (rIRC) safety [34], and concerns the observable's behaviour in the presence of multiple emissions. Essentially it involves two requirements: (a) if one scales all emissions in some appropriately uniform manner towards the soft and collinear limit, then the observable should scale in the same manner; and (b) if one emission is soft relative to the others, and kept of fixed relative softness while scaling all the emissions, then in the scaling limit, the observable should not change if the soft emission is removed. Again, this condition holds trivially for the jet veto, as long as one uses a standard "inclusive" longitudinally invariant infrared and collinear-safe hadron collider jet algorithm (e.g. $k_{t}$ [28, 39], Cambridge/Aachen [40-42], anti- $\left.k_{t}[35]\right)$. It ensures that double-logarithmic terms exponentiate and that one can use an independent-emission type approximation in evaluating NLL terms.

The master formula in the CAESAR approach then tells us that the NLL resummed jet-vetoed cross section is given by

$$
\begin{gathered}
\Sigma_{\mathrm{NLL}}\left(p_{\mathrm{t}, \text { veto }}\right)=\int d x_{1} d x_{2} f\left(x_{1}, p_{\mathrm{t}, \text { veto }}\right) f\left(x_{2}, p_{\mathrm{t}, \text { veto }}\right) \delta\left(x_{1} x_{2} s-M_{B}^{2}\right)\left|\mathcal{M}_{B}\right|^{2} \\
\cdot \mathcal{F}\left(R_{B}^{\prime}\right) e^{-R_{B}\left(p_{\mathrm{t}, \text { veto }}\right)}
\end{gathered}
$$

where $\left|\mathcal{M}_{B}\right|^{2}$ is the full tree-level matrix element squared to produce a $\mathrm{Z}$ or a Higgs boson including the flux, and spin- and colour-averages; $e^{-R_{B}}$ is a Sudakov form factor, with $R_{B}$ a double-logarithmic function given respectively for the $Z$ and Higgs-boson cases by

$$
\begin{aligned}
& R_{Z}\left(p_{\mathrm{t}, \text { veto }}\right)=2 C_{F} \int_{p_{\mathrm{t}, \text { veto }}^{2}}^{M_{Z}^{2}} \frac{d k_{t}^{2}}{k_{t}^{2}} \frac{\alpha_{s}^{\mathrm{CMW}}\left(k_{t}\right)}{\pi}\left(\ln \frac{M_{Z}}{k_{t}}-\frac{3}{4}\right), \\
& R_{H}\left(p_{\mathrm{t}, \text { veto }}\right)=2 C_{A} \int_{p_{\mathrm{t}, \text { veto }}^{2}}^{M_{H}^{2}} \frac{d k_{t}^{2}}{k_{t}^{2}} \frac{\alpha_{s}^{\mathrm{CMW}}\left(k_{t}\right)}{\pi}\left(\ln \frac{M_{H}}{k_{t}}-\frac{11 C_{A}-4 T_{R} n_{f}}{12 C_{A}}\right),
\end{aligned}
$$

with $\alpha_{s}^{\mathrm{CMW}}\left(k_{t}\right)$ the coupling constant in the CMW scheme [43], evaluated at scale $k_{t}$. The quantity $R_{B}^{\prime}$ is equal to $d R_{B} / d \ln \left(M_{B} / p_{\text {t,veto }}\right)$ and to NLL accuracy it is simply $4 C \alpha_{s}\left(p_{\text {t,veto }}\right) / \pi \ln \left(M_{B} / p_{\text {t,veto }}\right)$. It enters in the function $\mathcal{F}\left(R_{B}^{\prime}\right)$, which provides the singlelogarithmic contributions associated with the observable's dependence on multiple emis- 
sions. As derived in [34] (and references therein), $\mathcal{F}\left(R_{B}^{\prime}\right)$ is given by

$$
\begin{aligned}
\mathcal{F}\left(R_{B}^{\prime}\right)=\lim _{\epsilon \rightarrow 0} \epsilon^{R_{B}^{\prime}} \sum_{m=0}^{\infty} \frac{1}{m !} & \left(\prod_{i=1}^{m} \sum_{\ell_{i}=1}^{2} \frac{R_{B}^{\prime}}{2} \int_{\epsilon}^{\infty} \frac{d \zeta_{i}}{\zeta_{i}} \int_{0}^{1} d \xi_{i} \int_{0}^{2 \pi} \frac{d \phi_{i}}{2 \pi}\right) \times \\
& \times \Theta\left(1-\lim _{\bar{v} \rightarrow 0} \frac{V\left(\{\tilde{p}\}, \kappa_{1}\left(\zeta_{1} \bar{v}\right), \ldots, \kappa_{m}\left(\zeta_{m} \bar{v}\right)\right)}{\bar{v}}\right)
\end{aligned}
$$

where the $\kappa_{i}\left(\zeta_{i} \bar{v}\right)$ are parametrised momenta with azimuthal angle $\phi_{i}$, rapidity $y_{i}=$ $(-1)^{\ell_{i}} \xi_{i} \ln 1 / \bar{v}$ and $k_{t i}=\zeta_{i} \bar{v} M_{B}$, with the hard incoming momenta $\{\tilde{p}\}$ adjusted to ensure momentum conservation. In evaluating $\mathcal{F}$, a key point is that in the limit $\bar{v} \rightarrow 0$ all emissions become widely separated in rapidity. Consequently the jet algorithm, independently of the jet radius $R$ and whether it's of the SISCone [44] or generalised- $k_{t}[28,35,39-42]$ type, clusters each particle into a separate jet. The leading jet will then be given by the hardest of all the emissions, so that

$$
\lim _{\bar{v} \rightarrow 0} \frac{V\left(\{\tilde{p}\}, \kappa_{1}\left(\zeta_{1} \bar{v}\right), \ldots, \kappa_{m}\left(\zeta_{m} \bar{v}\right)\right)}{\bar{v}}=\max \left(\zeta_{1}, \ldots, \zeta_{n}\right)
$$

It is then straightforward to show that $\mathcal{F}\left(R_{B}^{\prime}\right)=1$. This can be verified also numerically with CAESAR. The result $\mathcal{F}\left(R_{B}^{\prime}\right)=1$ has been found before also for the resummation of the jet-resolution parameter in the $e^{+} e^{-}$Cambridge algorithm [29].

The feature of the jet veto observable that causes $\mathcal{F}\left(R_{B}^{\prime}\right)=1$ has consequences for the resummation beyond NLL. In particular certain key observable-dependent pieces of the NNLL resummation were outlined in the appendices of [34]. These are discussed further in appendix A. For the remainder of the main part of the article, however, we restrict our attention to NLL resummation.

In what follows below, rather than using eq. (3.3) directly as written, we shall reduce it to a form where the exponent involves just LL and NLL terms, expressed in terms of a $\operatorname{logarithm} L=\ln Q / p_{\text {t,veto }}$, with $Q$ the hard scale with respect to which the resummation is defined, as well as the $\overline{\mathrm{MS}}$ coupling $\alpha_{s}\left(\mu_{R}\right)$ at a given renormalisation scale $\mu_{R}$ and PDFs with a hard factorisation scale $\mu_{F}$. It is to be understood that $Q, \mu_{R}$ and $\mu_{F}$ will all be taken of order $M_{B}$. Varying them in the neighbourhood of $M_{B}$ will allow us to probe the impact of subleading terms. In practice, this is accomplished by expressing the CMW coupling in terms of the $\overline{\mathrm{MS}}$ coupling

$$
\alpha_{s}^{\mathrm{CMW}}=\alpha_{s}\left(1+K \frac{\alpha_{s}}{2 \pi}\right), \quad K=C_{A}\left(\frac{67}{18}-\frac{\pi^{2}}{6}\right)-\frac{5}{9} n_{f}
$$

and using for $\alpha_{s}\left(k_{t}\right)$ the following expression

$$
\alpha_{s}\left(k_{t}\right)=\frac{\alpha_{s}\left(\mu_{R}\right)}{1-2 \rho}\left(1-\alpha_{s}\left(\mu_{R}\right) \frac{\beta_{1}}{\beta_{0}} \frac{\ln (1-2 \rho)}{1-2 \rho}\right), \quad \rho \equiv \alpha_{s}\left(\mu_{R}\right) \beta_{0} \ln \frac{\mu_{R}}{k_{t}},
$$

with

$$
\beta_{0}=\frac{11 C_{A}-2 n_{f}}{12 \pi} \quad \beta_{1}=\frac{17 C_{A}^{2}-5 C_{A} n_{f}-3 C_{F} n_{f}}{24 \pi^{2}} .
$$


A closed result for the NLL resummed result can then be obtained by substituting eqs. (3.6), (3.7) into eq. (3.3):

$$
\begin{array}{rl}
\Sigma_{\mathrm{NLL}}\left(p_{\mathrm{t}, \text { veto }}\right)=\int d x_{1} d x_{2} & f\left(x_{1}, \mu_{F} e^{-L}\right) f\left(x_{2}, \mu_{F} e^{-L}\right) \delta\left(x_{1} x_{2} s-M_{B}^{2}\right)\left|\mathcal{M}_{B}\right|^{2} \\
\cdot & \exp \left[L g_{1}\left(\alpha_{s}\left(\mu_{R}\right) L\right)+g_{2}\left(\alpha_{s}\left(\mu_{R}\right) L\right)\right],
\end{array}
$$

with

$$
\begin{aligned}
g_{1}\left(\alpha_{s} L\right)=\frac{2 C}{\pi \beta_{0}}\left(1+\frac{\ln (1-2 \lambda)}{2 \lambda}\right), \\
g_{2}\left(\alpha_{s} L\right)=\frac{2 C}{\pi \beta_{0}}\left[-\left(\frac{K}{4 \pi \beta_{0}}+\ln \frac{\mu_{R}}{M_{B}}\right)\left(\ln (1-2 \lambda)+\frac{2 \lambda}{1-2 \lambda}\right)+B \ln (1-2 \lambda)\right. \\
\left.+\frac{\beta_{1}}{2 \beta_{0}^{2}}\left(\frac{1}{2} \ln ^{2}(1-2 \lambda)+\frac{\ln (1-2 \lambda)+2 \lambda}{1-2 \lambda}\right)-\ln \frac{M_{B}}{Q} \frac{2 \lambda}{1-2 \lambda}\right],
\end{aligned}
$$

where, for Higgs production $C=C_{A}$ and $B=-\left(11 C_{A}-2 n_{f}\right) /\left(12 C_{A}\right)$, whilst for DrellYan production, $C=C_{F}$ and $B=-3 / 4$, and $\lambda=\alpha_{s}\left(\mu_{R}\right) \beta_{0} L$. This form has the property that in the exponent there are only LL and NLL terms, whereas eq. (3.3) effectively also contained some higher-order contributions. Note also that the use in eq. (3.9) of $\mu_{F} e^{-L}$ rather than $p_{\text {t,veto }}$ in (3.3) corresponds to a NNLL difference.

One advantage of formulating the resummed result in terms of the variable $L$ is that it is then straightforward to adapt the resummation so as to ensure a sensible behaviour even for $p_{\mathrm{t}, \text { veto }} \gtrsim M_{B}$. This is done by "modifying" the logarithm,

$$
L \rightarrow \widetilde{L} \equiv \frac{1}{p} \ln \left(\left(\frac{Q}{p_{\mathrm{t}, \text { veto }}}\right)^{p}-\left(\frac{Q}{p_{\mathrm{t}, \text { veto }}^{\text {Max }}}\right)^{p}+1\right)
$$

where $p_{\mathrm{t} \text {,veto }}^{\mathrm{Max}}$ is the largest physically accessible jet momentum. For $Q \ll p_{\mathrm{t} \text {,veto }} \ll p_{\mathrm{t} \text {,veto }}^{\mathrm{Max}}$, $\widetilde{L} \sim\left(Q / p_{\text {t,veto }}\right)^{p} / p$ and thus $p$ can be chosen so as to ensure that resummation effects vanish quickly above the scale $Q$. In practice we choose $p=5$. This should help limit artifacts that could arise with the matching procedure introduced later on in this article.

The above way of formulating the resummation follows in the footsteps of [45], and includes refinements proposed in [46]. One of these refinements is that we allow $Q \neq M_{B}$, which accounts for the $\mathcal{O}(1)$ additive freedom in defining $L$ and the point where the resummation sets in. It plays an important role in uncertainty estimates.

For completeness we explicitly write the final form we use for the resummation cross section as $\widetilde{\Sigma}$, which is simply eq. (3.9) with $L$ replaced by $\widetilde{L}$ :

$$
\begin{gathered}
\widetilde{\Sigma}_{\mathrm{NLL}}\left(p_{\mathrm{t}, \text { veto }}\right)=\int d x_{1} d x_{2} f\left(x_{1}, \mu_{F} e^{-\widetilde{L}}\right) f\left(x_{2}, \mu_{F} e^{-\widetilde{L}}\right) \delta\left(x_{1} x_{2} s-M_{B}^{2}\right)\left|\mathcal{M}_{B}\right|^{2} \\
\cdot \exp \left[\widetilde{L} g_{1}\left(\alpha_{s}\left(\mu_{R}\right) \widetilde{L}\right)+g_{2}\left(\alpha_{s}\left(\mu_{R}\right) \widetilde{L}\right)\right]
\end{gathered}
$$

Figure 1 illustrates the resummed results for the Higgs-boson and $Z$-production cases. It shows the efficiency defined as

$$
\epsilon_{\mathrm{NLL}}\left(p_{\mathrm{t}, \text { veto }}\right)=\frac{\widetilde{\Sigma}_{\mathrm{NLL}}\left(p_{\mathrm{t}, \text { veto }}\right)}{\widetilde{\Sigma}_{\mathrm{NLL}}\left(p_{\mathrm{t}, \text { veto }}^{\operatorname{Max}}\right)} .
$$



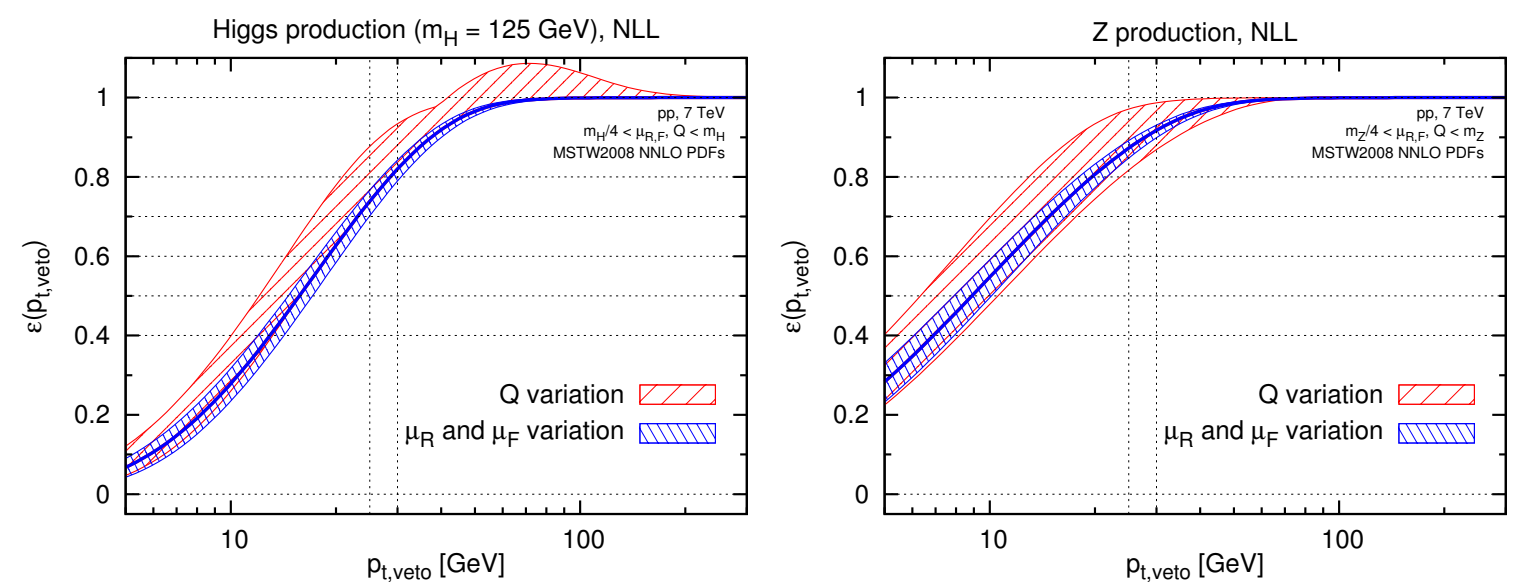

Figure 1. NLL resummed result for the jet-veto efficiency in Higgs (left) and $Z$-boson (right) production. The predictions are for $p p$ collisions at a centre of mass energy of $7 \mathrm{TeV}$ and use MSTW2008 NNLO PDFs [47]. For Higgs production we use the large $m_{\text {top }}$ approximation.

The vertical dashed lines mark the values of the jet-veto thresholds used by ATLAS and CMS experiments, of $25 \mathrm{GeV}$ and $30 \mathrm{GeV}$, respectively. The thick solid (blue) line corresponds to our central scale choice $\mu_{R}=\mu_{F}=Q=M_{B} / 2$. The finely hashed (blue) band around it corresponds to the envelope of results obtained when examining an independent variation of the renormalisation and factorisation scales with a constraint on their maximal ratio:

$$
\mu_{R}=\left\{\frac{M_{B}}{4}, \frac{M_{B}}{2}, M_{B}\right\}, \quad \mu_{F}=\left\{\frac{M_{B}}{4}, \frac{M_{B}}{2}, M_{B}\right\}, \quad \frac{1}{2} \leq \frac{\mu_{R}}{\mu_{F}} \leq 2 .
$$

The resulting uncertainty is relatively modest. More significant is the uncertainty associated with the variation of $Q$, shown by the widely-hashed (red) band, corresponding to the envelope of the choices $Q=\left\{M_{B} / 4, M_{B} / 2, M_{B}\right\}$ (all with $\mu_{R}=\mu_{F}=M_{B} / 2$ ). This variation probes the impact of unknown NNLL terms. In the case of Higgs production, one sees that the band even goes almost $10 \%$ above 1 . This kind of behaviour is common in NLL resummation and can be traced back to the $B$ term in eq. (3.10b) or equivalently the second, negative term in the round brackets of eq. (3.3c). The problem is less severe in the $Z$-production case, because of a cancellation due to PDF effects. These kinds of artifacts are one of the reasons why it is important to "match" resummations with fixed order predictions, especially in the region of moderate to large $p_{t}$ vetoes. In the next section we will therefore consider the structure of the fixed-order cross sections, and subsequently proceed to introduce our matching prescriptions and to examine the results.

\section{Jet-veto at fixed order}

The state-of-the-art of fixed-order predictions for fully differential differential partonic Higgs-boson and $Z$-boson cross sections is NNLO, i.e. the calculation of $\Sigma_{2}\left(p_{\text {t,veto }}\right)$ and $\sigma_{2}$, with tools like FEHIP [13] and HNNLO [14, 15] for Higgs productions, and FEWZ [48] 
and DYNNLO [49] for $\mathrm{Z}$ production. For the purpose of determining the jet-veto cross section, it is however also possible (and sometimes numerically cheaper) to compute only $\sigma_{2}$ with these NNLO tools (or from the inclusive results [50-53]), and obtain $\Sigma_{1}\left(p_{\text {t,veto }}\right)$ and $\Sigma_{2}\left(p_{\mathrm{t}, \mathrm{veto}}\right)$ from the relation

$$
\Sigma_{i}\left(p_{\mathrm{t}, \text { veto }}\right)=\sigma_{i}+\bar{\Sigma}_{i}\left(p_{\mathrm{t}, \text { veto }}\right), \quad \bar{\Sigma}_{i}\left(p_{\mathrm{t}, \text { veto }}\right)=-\int_{p_{\mathrm{t}, \text { veto }}}^{\infty} d p_{\mathrm{t}} \frac{d \Sigma_{i}\left(p_{\mathrm{t}}\right)}{d p_{\mathrm{t}}} .
$$

The differential distributions $d \bar{\Sigma}_{1} / d p_{\mathrm{t}}$ and $d \bar{\Sigma}_{2} / d p_{\mathrm{t}}$ can be computed from the boson+jet cross sections at LO and NLO respectively, e.g. using MCFM [19, 20, 54]. We recall that, throughout, we use the large $m_{\text {top }}$ approximation for Higgs production.

\subsection{Prescriptions for the efficiency}

There is little ambiguity in the definition of the fixed order results for the total and jetvetoed cross-sections, with the only freedom being, as usual, in the choice or renormalisation and factorisation scale. However, given the expressions of $\Sigma$ and $\sigma$ at a given perturbative order, there is some additional freedom in the way one computes the jet-veto efficiency. For instance, at NNLO the efficiency can be defined as

$$
\epsilon^{(a)}\left(p_{\mathrm{t}, \text { veto }}\right) \equiv \frac{\Sigma_{0}\left(p_{\mathrm{t}, \text { veto }}\right)+\Sigma_{1}\left(p_{\mathrm{t}, \mathrm{veto}}\right)+\Sigma_{2}\left(p_{\mathrm{t}, \mathrm{veto}}\right)}{\sigma_{0}+\sigma_{1}+\sigma_{2}},
$$

but the following expressions are equally valid at NNLO,

$$
\begin{aligned}
& \epsilon^{(b)}\left(p_{\mathrm{t}, \text { veto }}\right) \equiv \frac{\Sigma_{0}\left(p_{\mathrm{t}, \text { veto }}\right)+\Sigma_{1}\left(p_{\mathrm{t}, \text { veto }}\right)+\bar{\Sigma}_{2}\left(p_{\mathrm{t}, \mathrm{veto}}\right)}{\sigma_{0}+\sigma_{1}}, \\
& \epsilon^{(c)}\left(p_{\mathrm{t}, \text { veto }}\right) \equiv 1+\frac{\bar{\Sigma}_{1}\left(p_{\mathrm{t}, \mathrm{veto}}\right)}{\sigma_{0}}+\left(\frac{\bar{\Sigma}_{2}\left(p_{\mathrm{t}, \mathrm{veto}}\right)}{\sigma_{0}}-\frac{\sigma_{1}}{\sigma_{0}^{2}} \bar{\Sigma}_{1}\left(p_{\mathrm{t}, \text { veto }}\right)\right),
\end{aligned}
$$

since they differ relative to eq. (4.2a) only by terms $\mathcal{O}\left(\alpha_{s}^{3}\right)$, which are not under control.

Option $(a)$ is the most widely used, and may appear at first sight to be the most natural, since one keeps as many terms as possible both in the numerator and denominator. However, option (b) can be motivated as follows: since the zeroth order term of $\epsilon\left(p_{\mathrm{t}, \text { veto }}\right)$ is equal to 1 , it is really only $1-\epsilon\left(p_{\mathrm{t}, \text { veto }}\right)$ that has a non-trivial perturbative series, given by the ratio of the inclusive 1 -jet cross section above $p_{\mathrm{t} \text {,veto }}, \sigma_{1 \text {-jet }}^{\mathrm{NLO}}\left(p_{\mathrm{t} \text {,veto }}\right)$, to the total cross section. Insofar as the 1 -jet cross section is known only to NLO, in taking the ratio to the total cross section one can argue that one should also use NLO for the latter, i.e.

$$
\epsilon\left(p_{\mathrm{t}, \mathrm{veto}}\right)=1-\frac{\sigma_{1-\mathrm{jet}}^{\mathrm{NLO}}\left(p_{\mathrm{t}, \mathrm{veto}}\right)}{\sigma_{0}+\sigma_{1}} .
$$

It is straightforward to verify that this then leads to eq. (4.2b). This procedure also coincides with the one adopted in event-shape studies in DIS and hadron-hadron collisions ( $\sigma_{2}$ is not even known in the latter case). Option $(c)$ is also well motivated, since it is a strict fixed order expansion of the ratio, so no uncontrolled terms beyond NNLO are included. This is the prescription that is usually adopted in $e^{+} e^{-}$event-shape and jet-rate studies. 

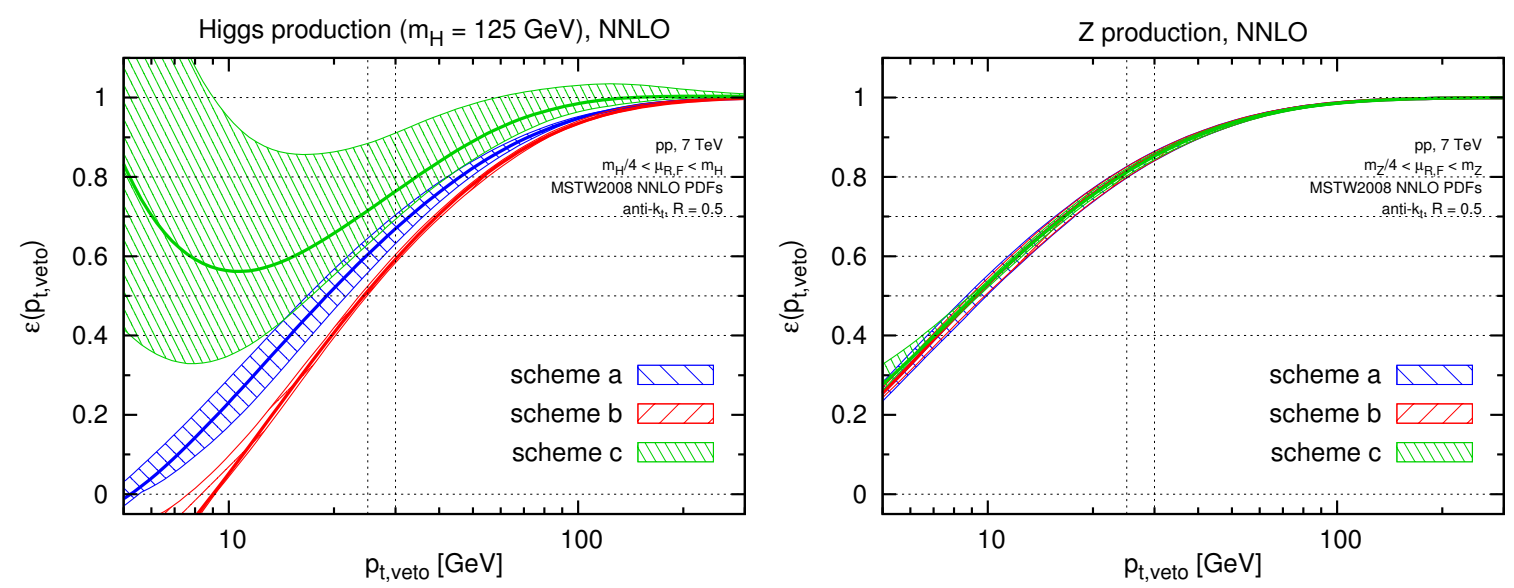

Figure 2. Jet-veto efficiency for Higgs (left) and $Z$-boson production (right) using three different prescriptions for the NNLO expansion, see eqs. (4.2a)-(4.2c). For each prescription, the thick solid line corresponds to the result obtained with $\mu_{R}=\mu_{F}=M_{H / Z} / 2$, while the band shows the scale uncertainty as obtained with the choices of eq. (3.14).

While other possibilities are also equally valid, the above three schemes capture a substantial part of the freedom that one has in writing the series. The size of the differences between them is one way to estimate the associated theoretical uncertainty and goes beyond the usual variation of scales.

\subsection{Numerical results}

Figure 2 shows the NNLO results for the jet-veto efficiency in the 3 schemes discussed above. Each scheme is displayed as a band corresponding to the envelope of the scale variations as in eq. (3.14), together with a solid line for the prediction with the central scale choice.

In the case of Higgs production (left-hand plot) the bands barely overlap and, in the region of interest, $p_{\mathrm{t} \text {,veto }} \sim 25-30 \mathrm{GeV}$, the three predictions differ considerably, with the bands spanning the range from 0.6 to 0.9 and the central values from 0.6 to 0.77 (for

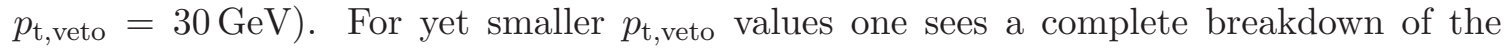
fixed-order predictions, with the efficiencies ranging from below 0 to above 1 . Curiously, scheme-c misbehaves also at large $p_{\text {t,veto }} \sim M_{H}$, where it leads to an efficiency larger than 1 .

In contrast, for $Z$ production (right-hand plot), the situation is much more stable, with all three schemes overlapping and remaining sensible-looking down even to low $p_{\text {t,veto }}$.

The large differences between the Higgs and $Z$-boson cases call for an explanation. One consideration is that the $g g$ initial state that is relevant for Higgs production implies $C_{A}=3$ colour factors rather than $C_{F}=\frac{4}{3}$ colour factors. This inevitably worsens the perturbative convergence. The larger colour factor is however not the only culprit. Table 1 shows the total cross sections for Higgs and $Z$ production calculated at different orders, as well as the coefficients $c_{i}$ of the perturbative expansion normalised to $\sigma_{0}$ at the central scale choice, i.e. $c_{i}=\sigma_{i} /\left(\sigma_{0} \alpha_{s}^{i}\right)$ such that the cross section reads

$$
\sigma=\sigma_{0}\left(1+c_{1} \alpha_{s}+c_{2} \alpha_{s}^{2}+\ldots\right) .
$$




\begin{tabular}{|cccccc|}
\hline & $\mathrm{LO}$ & $\mathrm{NLO}$ & $\mathrm{NNLO}$ & $c_{1}$ & $c_{2}$ \\
\hline $\mathrm{H}[\mathrm{pb}]$ & $5.40_{-1.02}^{+1.43}$ & $11.96_{-1.95}^{+2.63}$ & $14.7_{-1.4}^{+1.2}$ & 9.78 & 33.5 \\
$\mathrm{Z}[\mathrm{nb}]$ & $22.85_{-2.40}^{+2.07}$ & $28.6_{-1.2}^{+0.8}$ & $28.6_{-0.4}^{+0.4}$ & 1.94 & 0.0 \\
\hline
\end{tabular}

Table 1. Cross-sections for $\mathrm{Z}$ and Higgs (large $m_{\text {top }}$ approximation) production at various orders in perturbation theory. The central value corresponds to the default scale, $\mu_{R}=\mu_{F}=M_{B} / 2$, $M_{B}=M_{Z}$ or $M_{H}$, the error denotes the scale variation when $\mu_{R}$ and $\mu_{F}$ are varied independently by a factor two around the central value, with the constraint that $\mu_{R} / 2 \leq \mu_{F} \leq 2 \mu_{R}$, i.e. eq. (3.14). Also shown are the expansion coefficients $c_{i}$ of the cross-sections for the central scale $\mu_{0}=M_{B} / 2$, $\sigma_{i}=\sigma_{0} c_{i} \alpha_{s}^{i}\left(\mu_{0}\right)$. The results were obtained using the HNNLO [14, 15] and DYNNLO [49] programs, with MSTW2008 NNLO PDFs and $\alpha_{s}\left(M_{Z}\right)=0.11707$, and a centre-of-mass energy of $7 \mathrm{TeV}$.

\begin{tabular}{|cccccc|}
\hline & LO & NLO & NNLO & $c_{1}$ & $c_{2}$ \\
\hline H $[\mathrm{pb}]$ & $5.40_{-1.02}^{+1.43}$ & $8.99_{-1.16}^{+1.33}$ & $9.88_{-0.55}^{+0.27}$ & 5.36 & 10.6 \\
Z $[\mathrm{nb}]$ & $22.85_{-2.40}^{+2.07}$ & $25.54_{-1.02}^{+0.64}$ & $24.5_{-0.7}^{+0.6}$ & 0.90 & -2.7 \\
\hline
\end{tabular}

Table 2. Same as table 1 but with a jet-veto of $30 \mathrm{GeV}$, based on the anti- $k_{t}$ algorithm with $R=0.5$. The LO cross-section is equal to that without a veto. The results have been determined by subtracting $H+1$-jet and $Z+1$-jet cross sections, obtained with MCFM, from the inclusive cross sections obtained with DYNNLO and HNNLO, though we could equally well have used DYNNLO and HNNLO directly.

The difference between schemes $a$ and $b$ is in the choice of whether the 1-jet cross section is normalised to the NLO or NNLO total cross section. The NNLO term brings a correction of order $20 \%$ for Higgs production and this is reflected in the efficiencies; for $Z$ production, $\sigma_{2}$ is essentially zero and so schemes $a$ and $b$ give identical results. As concerns scheme $c$, one is additionally affected by the very large size of $\sigma_{1}$ in the Higgs case $[55,56]$.

Table 2 shows corresponding cross section results with a jet-veto of $30 \mathrm{GeV}$. The $Z$ production case maintains its reasonable convergence. Interestingly, the vetoed Higgs cross section now also appears to have a convergence that is significantly improved with respect to the total cross section. As has been argued by others [57], this apparent improvement in convergence can be interpreted as an artifact of cancellations between two independent classes of large corrections: those that lead to the poor convergence of the total cross section and those associated with the Sudakov suppression for small jet vetoes (enhanced compared to the $\mathrm{Z}$ case due to the larger colour factors for Higgs production). The presence of such artificial cancellations in the cross section is one of the reasons that we prefer to consider the jet veto efficiency. Furthermore, as we have seen, the use of the jet veto efficiency provides additional handles to estimate the perturbative uncertainty through the three schemes discussed above.

Given that we have indicated that the uncertainties on the Higgs jet-veto efficiency are associated with the poor convergence of the total Higgs cross section, some comments are due concerning discussions in the literature on our knowledge of the total Higgs cross section. On one hand there is work that aims to account for threshold and other enhanced 
terms beyond NNLO [58-61], while other work has suggested that there is a need to revisit, and perhaps be more conservative in, estimates of the uncertainties on the total cross section [62]. Regarding improvements of the total cross section, while in general these have the potential to be highly valuable, a more accurate evaluation of the total cross section is, we believe, likely to provide little improvement for the jet-veto efficiency: indeed, our results indicate that there are potentially large missing $\mathcal{O}\left(\alpha_{s}^{3}\right)$ corrections to the jet veto efficiency and it is only through an evaluation of the NNLO $\mathrm{H}+1$-jet rate that these could be fully constrained.

\section{$5 \quad$ Prescription for estimating total uncertainties}

From now onwards, for the purpose of quoting final efficiency estimates, we will need a prescription for combining different sources of uncertainty. We shall adopt the envelope method, used for example in [63] as well as in many other works on resummation. This method takes the envelope of the curves obtained from each of several different sources of uncertainty estimate. The logic of the scheme is that to avoid double counting uncertainties, at most one source should be probed at a time. Thus here at fixed order we would take the envelope that results from the scale variation band from one main efficiency prescription (scheme $a$ ) and the central values of each of the two alternative efficiency prescriptions ( $b$ and $c$ ). As has been found in [64], this leads to an uncertainty estimate that is quite close to the alternative fixed-order prescription proposed in [57], which varies the scale choice independently in zero, one and two-jet bins.

In addition to estimating the uncertainty on the veto efficiency, it is also important to be able to estimate the uncertainty on the final vetoed cross section. The prescription that we propose is to treat the efficiency uncertainty as being uncorrelated with the uncertainty on the total cross section. This can be justified on the following grounds: the total cross section uncertainty is associated with our ignorance of the NNNLO corrections to its perturbative series. On the other hand the uncertainties in the efficiency are related to higher-order Sudakov type terms and to the way in which we treat the known NNLO cross-section contributions in calculating the efficiency. These are sufficiently different in origin that we believe that it is reasonable to treat them as uncorrelated.

\section{Matching NLL and NNLO results}

To obtain predictions for the jet veto efficiency that include the advantages of both resummed and fixed order results, we combine them with the help of a matching procedure. Below, we first briefly recall the various requirements that matching should satisfy, then list the matching prescriptions that we adopt, and finally discuss the matched results.

\subsection{Matching prescriptions}

As is well-known there are various ways in which one can match resummed and fixed order calculations. Since we match the resummation to NNLO exact results, the matching procedure should satisfy the following three requirements: 
1. The matched result should be correct up to NLL terms in the exponent and the expanded matched result should be correct up to and including $\mathcal{O}\left(\alpha_{s}^{n} L^{2 n-2}\right)$ terms.

2. The expanded matched result should coincide with the fixed order result up to and including the NNLO terms.

3. The jet-veto efficiency should tend to one at the maximum allowed jet transverse momentum $p_{\mathrm{t}, \mathrm{veto}}^{\mathrm{Max}}$, and the corresponding differential distribution $d \epsilon\left(p_{\mathrm{t}, \mathrm{veto}}\right) / d p_{\mathrm{t} \text {, veto }}$ should vanish at that point

$$
\epsilon\left(p_{\mathrm{t}, \text { veto }}\right)=1 \quad \text { and } \quad \frac{d \epsilon\left(p_{\mathrm{t}, \text { veto }}\right)}{d p_{\mathrm{t}, \text { veto }}}=0 \quad \text { for } \quad p_{\mathrm{t}, \text { veto }}=p_{\mathrm{t}, \text { veto }}^{\mathrm{Max}} .
$$

The use of the modified logarithm $\widetilde{L}$, defined in eq. (3.11) makes it relatively straightforward to fulfil this condition. Note that at the LHC, $p_{\mathrm{t}, \text { veto }}^{\mathrm{Max}}$ is much larger than the $p_{\text {t,veto }}$ values that will be of interest to us. Therefore, while we take care to enforce eq. (3.12), the terms involving $p_{\mathrm{t} \text {,veto }}^{\mathrm{Max}}$ will in practice largely be irrelevant. Nevertheless the use of the modified logarithm is important also because it ensures that the $\widetilde{L}$ that enters in eq. (3.12) approaches zero for $p_{\text {t,veto }} \gtrsim Q$.

Even with these conditions, there is some freedom in the matching procedure. This is closely related to the freedom we had in expressing the efficiencies. We will therefore consider three matching schemes, each of which is the counterpart of one of our fixed-order efficiency prescriptions, so as to facilitate the comparison with the NNLO results.

The first of our matching schemes is given by

$$
\begin{aligned}
& \Sigma_{\text {matched }}^{(a)}\left(p_{\mathrm{t}, \text { veto }}\right)= \\
& \begin{aligned}
\left(\frac{\widetilde{\Sigma}_{\mathrm{NLL}}\left(p_{\mathrm{t}, \text { veto }}\right)}{\sigma_{0}}\right)^{Z} & {\left[\sigma_{0}+\Sigma_{1}\left(p_{\mathrm{t}, \text { veto }}\right)+\Sigma_{2}\left(p_{\mathrm{t}, \text { veto }}\right)-Z\left(\widetilde{\Sigma}_{\mathrm{NLL}, 1}\left(p_{\mathrm{t}, \text { veto }}\right)+\widetilde{\Sigma}_{\mathrm{NLL}, 2}\left(p_{\mathrm{t}, \text { veto }}\right)\right)\right.} \\
& \left.-Z \frac{\widetilde{\Sigma}_{\mathrm{NLL}, 1}\left(p_{\mathrm{t}, \text { veto }}\right)}{\sigma_{0}}\left(\Sigma_{1}\left(p_{\mathrm{t}, \text { veto }}\right)-\frac{Z+1}{2} \widetilde{\Sigma}_{\mathrm{NLL}, 1}\left(p_{\mathrm{t}, \text { veto }}\right)\right)\right], \quad
\end{aligned}
\end{aligned}
$$

where $\widetilde{\Sigma}_{\mathrm{NLL}, \mathrm{i}}\left(p_{\mathrm{t}, \text { veto }}\right)$ is the term of $\mathcal{O}\left(\alpha_{s}^{i}\right)$ relative to $\sigma_{0}$ in the fixed-order expansion of $\widetilde{\Sigma}_{\mathrm{NLL}}$. The factor $Z=\left(1-\frac{p_{\mathrm{t} \text {,veto }}}{p_{\mathrm{t}, \text { veto }}}\right)$ is necessary to satisfy eq. $(6.1)$, but is largely irrelevant in practice. The corresponding jet-veto efficiency is

$$
\epsilon_{\text {matched }}^{(a)}\left(p_{\mathrm{t}, \mathrm{veto}}\right)=\frac{\Sigma_{\text {matched }}^{(a)}\left(p_{\mathrm{t}, \mathrm{veto}}\right)}{\Sigma_{\text {matched }}^{(a)}\left(p_{\mathrm{t}, \mathrm{veto}}^{\text {Max }}\right)}
$$

It is straightforward to verify that with this matching procedure $\epsilon_{\text {matched }}^{(a)}\left(p_{\text {t,veto }}\right)$ satisfies all three requirements listed at the beginning of this section. 
The second matching scheme is identical except that we replace $\Sigma_{2}\left(p_{\text {t,veto }}\right)$ with $\bar{\Sigma}_{2}\left(p_{\text {t,veto }}\right)$, in direct analogy with scheme $b$ used for the fixed order efficiency definition,

$$
\begin{aligned}
& \Sigma_{\text {matched }}^{(b)}\left(p_{\text {t,veto }}\right)= \\
& \left(\frac{\widetilde{\Sigma}_{\mathrm{NLL}}\left(p_{\mathrm{t}, \mathrm{veto}}\right)}{\sigma_{0}}\right)^{Z}\left[\sigma_{0}+\Sigma_{1}\left(p_{\mathrm{t}, \text { veto }}\right)+\bar{\Sigma}_{2}\left(p_{\mathrm{t}, \text { veto }}\right)-Z\left(\widetilde{\Sigma}_{\mathrm{NLL}, 1}\left(p_{\mathrm{t}, \text { veto }}\right)+\widetilde{\Sigma}_{\mathrm{NLL}, 2}\left(p_{\mathrm{t}, \text { veto }}\right)\right)\right. \\
& \left.-Z \frac{\widetilde{\Sigma}_{\mathrm{NLL}, 1}\left(p_{\mathrm{t}, \mathrm{veto}}\right)}{\sigma_{0}}\left(\Sigma_{1}\left(p_{\mathrm{t}, \mathrm{veto}}\right)-\frac{Z+1}{2} \widetilde{\Sigma}_{\mathrm{NLL}, 1}\left(p_{\mathrm{t}, \mathrm{veto}}\right)\right)\right]
\end{aligned}
$$

with

$$
\epsilon_{\text {matched }}^{(b)}\left(p_{\mathrm{t}, \mathrm{veto}}\right)=\frac{\Sigma_{\text {matched }}^{(b)}\left(p_{\mathrm{t}, \mathrm{veto}}\right)}{\Sigma_{\text {matched }}^{(b)}\left(p_{\mathrm{t}, \mathrm{veto}}^{\text {Max }}\right)} .
$$

The third scheme that we consider is formulated directly in terms of the jet-veto efficiency and is thus close in spirit to fixed-order scheme $c$ :

$$
\begin{aligned}
\epsilon_{\text {matched }}^{(c)}\left(p_{\mathrm{t}, \text { veto }}\right)= & \\
\left(\epsilon_{\mathrm{NLL}}\left(p_{\mathrm{t}, \text { veto }}\right)\right)^{Z} & {\left[1+\epsilon_{1}\left(p_{\mathrm{t}, \text { veto }}\right)+\epsilon_{2}\left(p_{\mathrm{t}, \text { veto }}\right)-Z\left(\epsilon_{\mathrm{NLL}, 1}\left(p_{\mathrm{t}, \text { veto }}\right)+\epsilon_{\mathrm{NLL}, 2}\left(p_{\mathrm{t}, \text { veto }}\right)\right)\right.} \\
& \left.-Z \epsilon_{\mathrm{NLL}, 1}\left(p_{\mathrm{t}, \text { veto }}\right)\left(\epsilon_{1}\left(p_{\mathrm{t}, \text { veto }}\right)-\frac{Z+1}{2} \epsilon_{\mathrm{NLL}, 1}\left(p_{\mathrm{t}, \text { veto }}\right)\right)\right]
\end{aligned}
$$

where, in accord with earlier definitions, we have

$$
\begin{aligned}
\epsilon_{\mathrm{NLL}}\left(p_{\mathrm{t}, \mathrm{veto}}\right) & \equiv \frac{\widetilde{\Sigma}_{\mathrm{NLL}}\left(p_{\mathrm{t}, \mathrm{veto}}\right)}{\sigma_{0}}, \\
\epsilon_{\mathrm{NLL}, i}\left(p_{\mathrm{t}, \mathrm{veto}}\right) & \equiv \frac{\tilde{\Sigma}_{\mathrm{NLL}, i}\left(p_{\mathrm{t}, \mathrm{veto}}\right)}{\sigma_{0}}, \\
\epsilon_{1}\left(p_{\mathrm{t}, \text { veto }}\right) & \equiv \frac{\bar{\Sigma}_{1}\left(p_{\mathrm{t}, \mathrm{veto}}\right)}{\sigma_{0}}, \\
\epsilon_{2}\left(p_{\mathrm{t}, \text { veto }}\right) & \equiv \frac{\bar{\Sigma}_{2}\left(p_{\mathrm{t}, \mathrm{veto}}\right)}{\sigma_{0}}-\frac{\sigma_{1} \bar{\Sigma}_{1}\left(p_{\mathrm{t}, \mathrm{veto}}\right)}{\sigma_{0}^{2}} .
\end{aligned}
$$

The three schemes differ in two respects: firstly they treat non-asymptotic terms (i.e. $p_{\text {t,veto }} \sim Q$ ) differently; secondly, they lead to different sets of subleading logarithms. In particular, schemes $a$ and $b$ differ at the level of $\mathrm{N}^{4} \mathrm{LL}$ terms, while scheme $c$ differs from both of them by $\mathrm{N}^{3} \mathrm{LL}$ terms. We recall that NNLL differences will be probed by scale variations.

The above three schemes all belong to the family of multiplicative matchings [46]. Other schemes exist. In particular we have also examined the log- $R$ scheme of ref. [45] and found that its results are contained within the band defined by the three schemes above.

\subsection{Matched results}

Figure 3 shows the NLL+NNLO matched results for each of the three schemes discussed above. Each band corresponds to the envelope of renormalisation, factorisation and $Q$ scale 

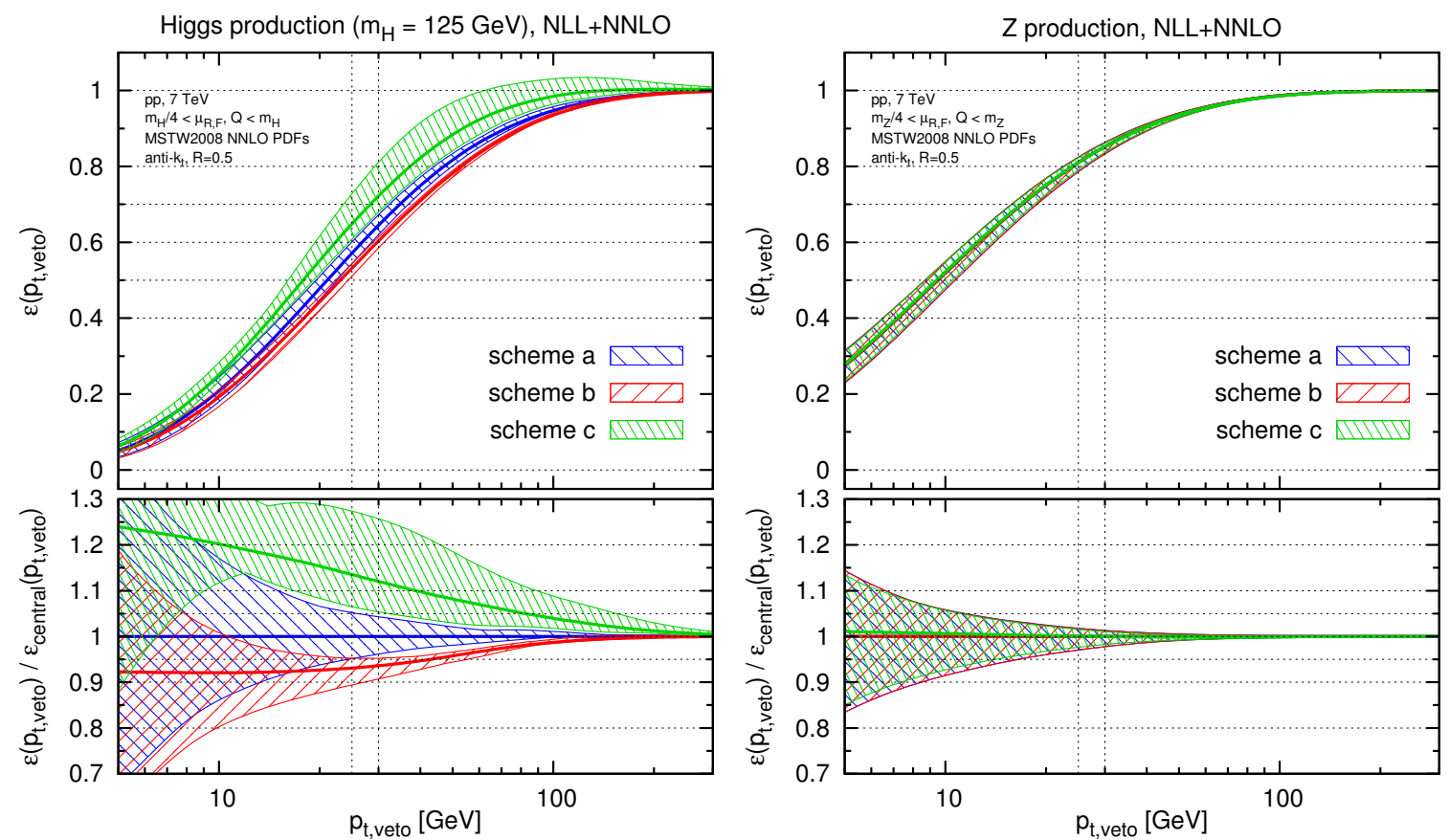

Figure 3. NLL+NNLO jet-veto efficiency for Higgs (left) and $Z$-boson production (right) using three different matching prescriptions. For each one, the thick solid line corresponds to the result obtained with $\mu_{R}=\mu_{F}=Q=M_{H / Z} / 2$, while the band shows the scale uncertainty as obtained from the envelope of the choices of eq. (3.14) and from $Q$-scale variation (taking $Q=\left\{M_{B} / 4, M_{B} / 2, M_{B}\right\}$ for $\mu_{R}=\mu_{F}=M_{H / Z} / 2$ ). The lower panels show the results normalised to the central scale choice for scheme $a$.

variation. Comparing to the pure resummed and fixed-order results of figures 1 and 2, one observes that for larger $p_{\mathrm{t} \text {,veto }}$, the results display the features of the fixed-order results, with similar uncertainties and, for scheme $c$, the same artifact of $\epsilon>1$ for certain scale choices. As in the NNLO case, for Higgs production the uncertainty bands from the three schemes mostly do not overlap, which serves to highlight the importance of the use of different schemes for probing uncertainties. For low $p_{\text {t,veto }}$ values, the results coincide with the pure resummation, though with uncertainty bands that are a little narrower, and that overlap even in the Higgs case. In the region of intermediate $p_{\text {t,veto }} \sim 25-30 \mathrm{GeV}$, the central value from the matched calculation is closer to that of the fixed-order results than to the resummed results, but with a slightly reduced uncertainty, indicating that resummation is at the edge of its validity in this region. Here too the bands from the different matching schemes fail to overlap in the Higgs case. Since the bands differ at most by NNNLL terms, this has implications for the degree of improvement that one might expect when extending the resummation from NLL to NNLL accuracy.

A direct comparison of the fixed-order and matched predictions is to be found in figure 4. Here the uncertainty envelopes encompass the full scheme $a$ band as well as the central values of the two other schemes. This follows the procedure outlined in section 5 and it provides the uncertainties that we shall use throughout the rest of the article. 

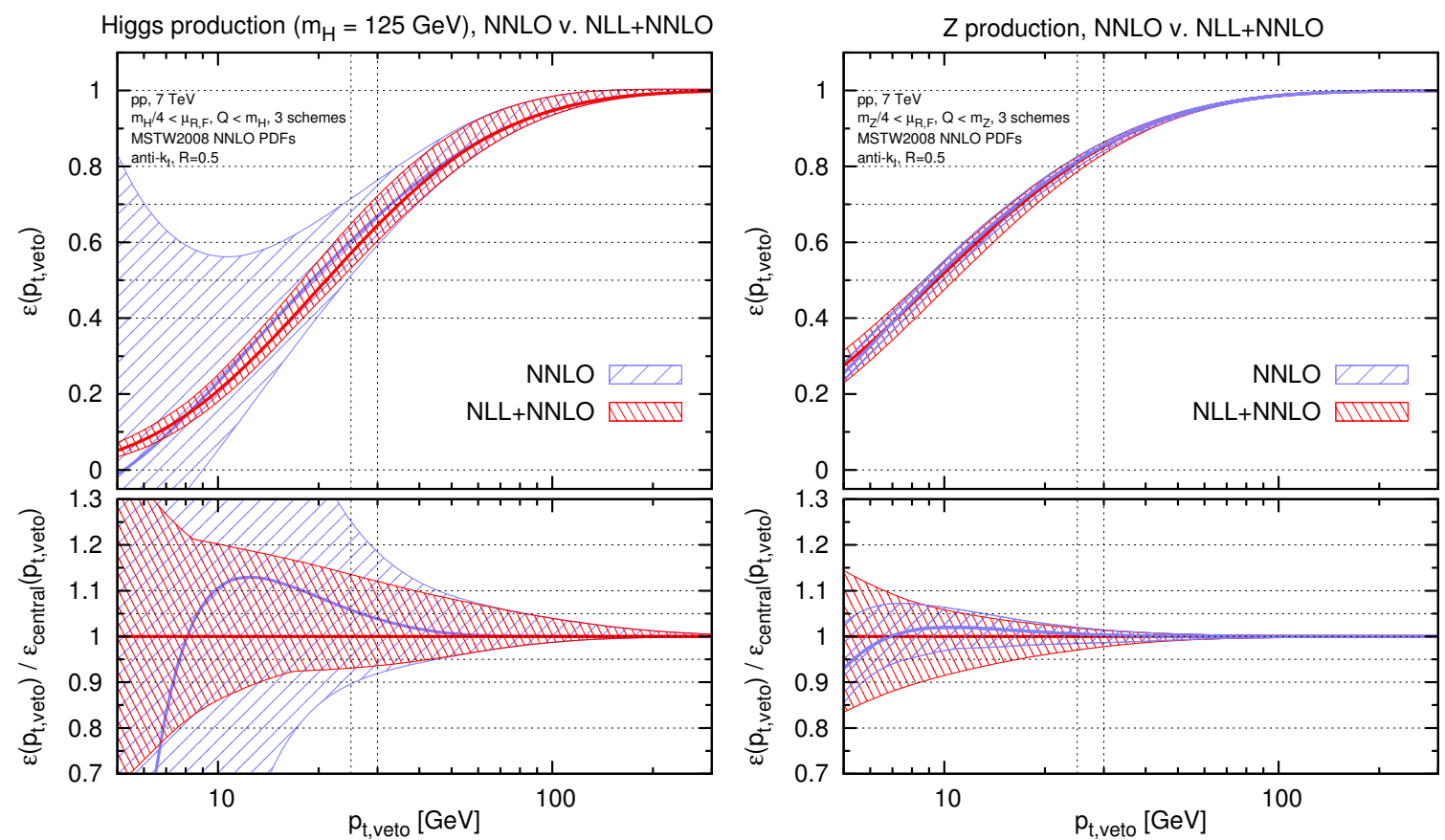

Figure 4. Comparison of fixed-order (NNLO) and matched resummed (NLL+NNLO) predictions for the jet veto efficiencies in Higgs (left) and $Z$ production (right). The uncertainties are those derived from the envelope method: for both fixed order and matched results they include renormalisation and factorisation scale uncertainties, as well as the scheme for defining the efficiency (or matching prescription). In the matched case, there is additionally the uncertainty from the variation of $Q$. The lower panels show the ratio of the results to the central matched prediction.

\begin{tabular}{|ccc|}
\multicolumn{3}{|c|}{ Higgs production $\left(M_{H}=125 \mathrm{GeV}\right)$} \\
\hline & NNLO & NLL+NNLO \\
\hline$p_{\text {t,veto }}=25 \mathrm{GeV}$ & $60_{-9}^{+11} \%$ & $57_{-4}^{+8} \%$ \\
$p_{\text {t,veto }}=30 \mathrm{GeV}$ & $67_{-8}^{+9_{\%}} \%$ & $64_{-4}^{+8} \%$ \\
\hline
\end{tabular}

\begin{tabular}{|c|c|c|}
\hline \multicolumn{3}{|c|}{$\mathrm{Z}$ production } \\
\hline & NNLO & $\mathrm{NLL}+\mathrm{NNLO}$ \\
\hline$p_{\mathrm{t}, \text { veto }}=25 \mathrm{GeV}$ & $81_{-2}^{+1} \%$ & $81_{-2}^{+1} \%$ \\
\hline$p_{\mathrm{t}, \text { veto }}=30 \mathrm{GeV}$ & $85_{-1}^{+1} \%$ & $85_{-2}^{+1} \%$ \\
\hline
\end{tabular}

Table 3. Jet veto efficiencies and their uncertainties at NNLO and NLL+NNLO, for the values of $p_{\mathrm{t}, \text { veto }}$ used by ATLAS and CMS, shown for the anti- $k_{t}$ algorithm with $R=0.5$, and based on MSTW2008 NNLO PDFs.

The efficiencies for the two jet-veto thresholds used by ATLAS and CMS, 25 and $30 \mathrm{GeV}$ respectively, are summarised in table 3. For Higgs production, one observes that the absolute efficiencies are about 3\% lower in the matched calculation as compared to the NNLO result (equivalent to a relative $5 \%$ reduction in the efficiency). The uncertainties are somewhat more asymmetric in the matched calculation and in particular the uncertainty towards lower efficiencies is reduced by about a factor of two. For $Z$-boson production, the uncertainties with matching are the same or larger as those of the pure NNLO result. This surprising result may be because the resummation explicitly involves the running of the coupling and thus, for low $p_{\mathrm{t}, \mathrm{veto}}$, directly probes the uncertainties associated with a 

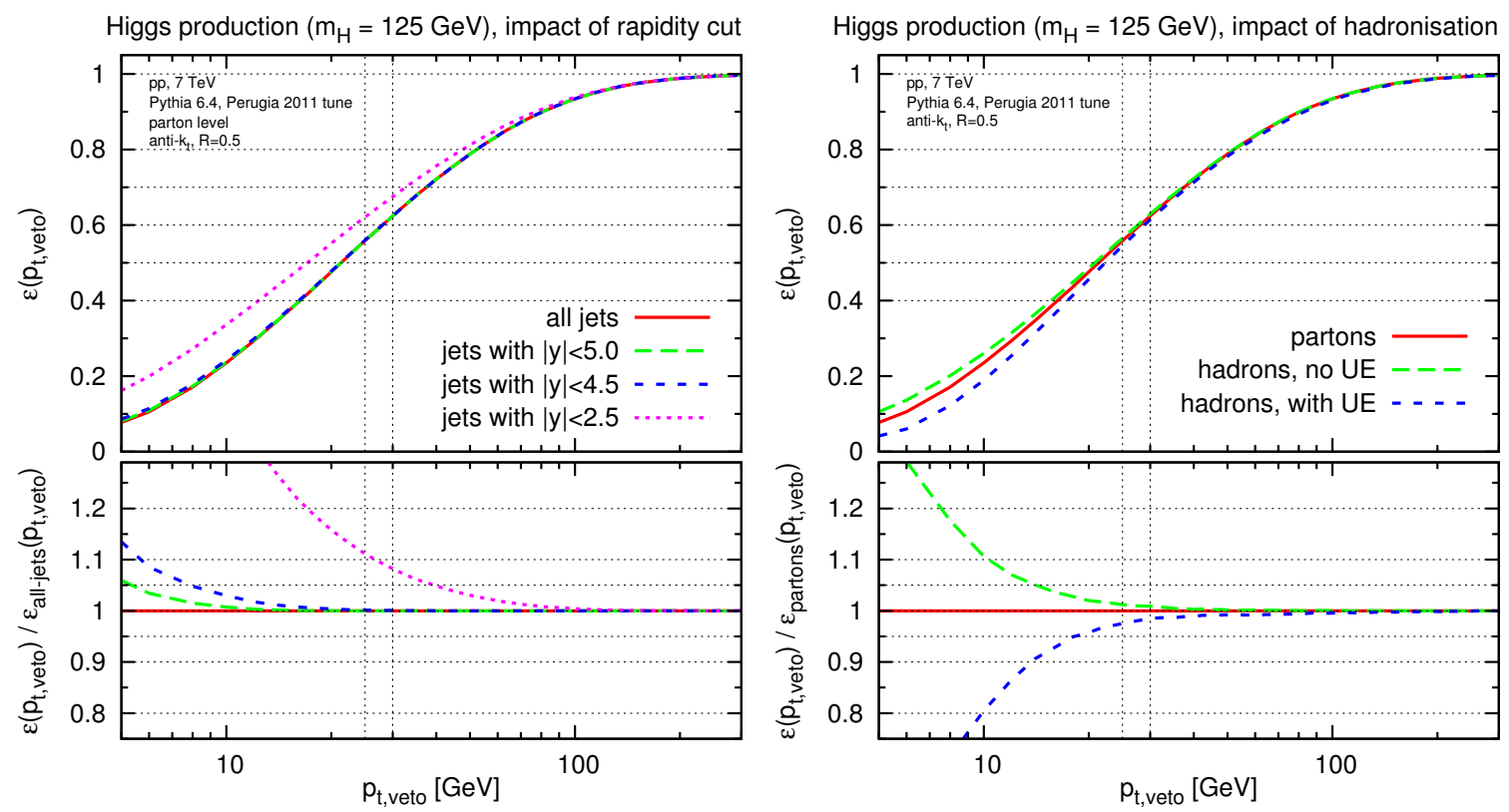

Figure 5. Left: impact of a finite rapidity acceptance for jets on the jet-veto efficiency, as calculated with PYTHIA 6.425. Right: impact of hadronisation and underlying event on the jet-veto efficiency. See text for further details.

perturbative expansion whose coupling constant is somewhat larger than the $\alpha_{s}\left(M_{Z} / 2\right)$ that appears in the NNLO calculation.

\section{Comparisons to other calculations}

In this section we will complement our resummed matched study so far with information from event generators and analytical boson- $p_{t}$ resummations. For brevity we concentrate on the case of Higgs production, using $M_{H}=125 \mathrm{GeV}$ throughout.

\subsection{Effects beyond the scope of matched calculations}

The matched calculation that we have performed applies to partons and assumes infinite detector acceptance. Experiments, however, measure hadrons, including the underlying event, and have limited acceptance, notably for the rapidity of the jets.

To investigate these two effects we have taken events generated by PYTHIA 6.4 [65] with the Perugia 2011 tune [66]. Jet clustering for the results in this section is performed with FastJet [67,68]. Figure 5 (left) shows the impact of considering jets only within some finite rapidity acceptance. One sees that for the choices used by ATLAS and CMS, $|y|<4.5$ and $y<|5.0|$ respectively, the veto efficiencies are almost identical to those with full acceptance in the practically relevant range of $p_{\text {t,veto }}$. We have confirmed that this pattern holds also in fixed-order calculations. In contrast, if one applies a jet veto only in a more restricted rapidity region, e.g. $|y|<2.5$, there are substantial differences.

The right-hand plots of figure 5 show the impact of non-perturbative effects. While not entirely negligible, both hadronisation and the underlying event have an impact that is 

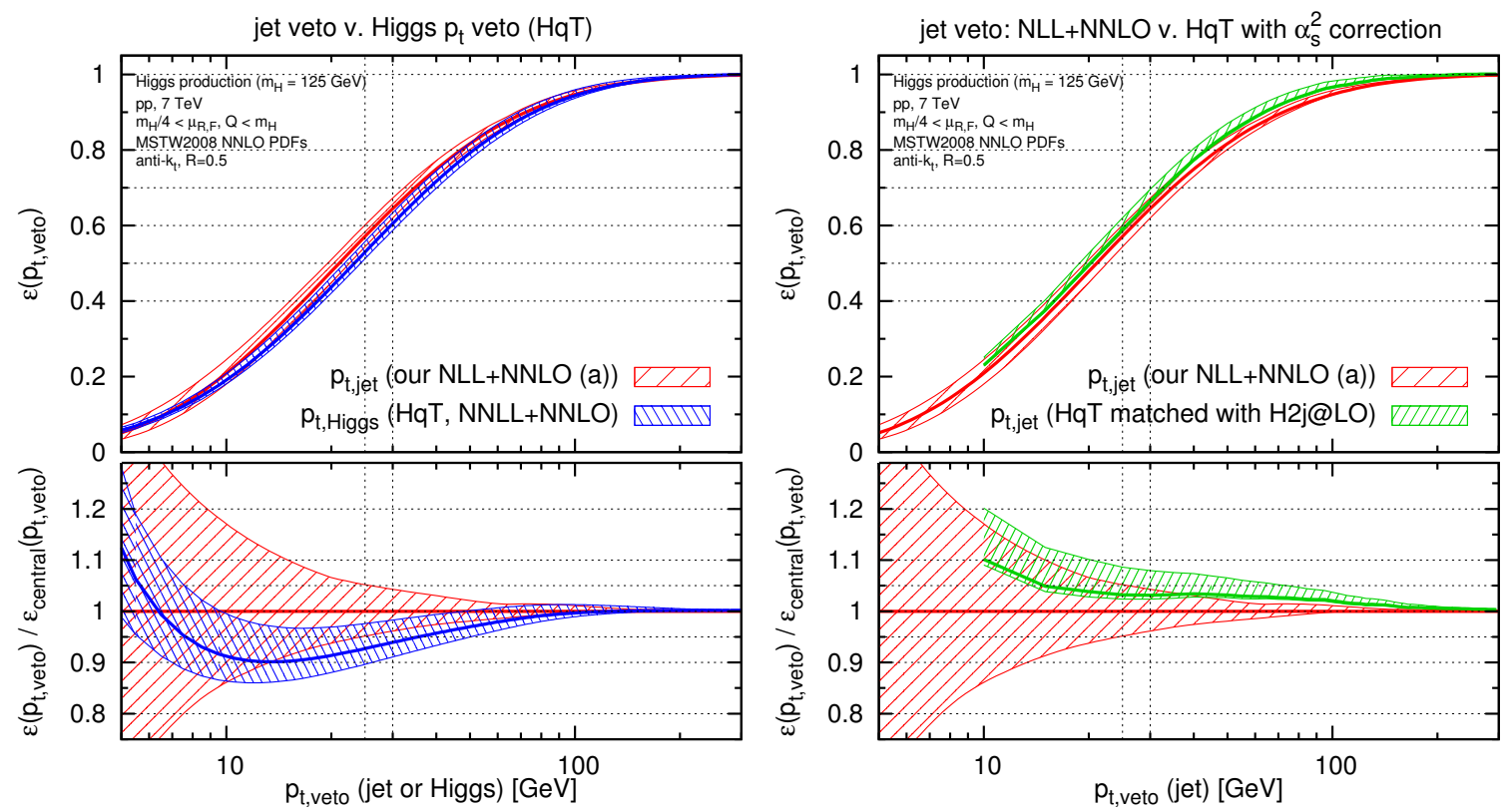

Figure 6. Left: comparison of our result for the jet veto efficiency with the calculation of a Higgsboson transverse momentum veto efficiency obtained with HqT. For the purpose of the comparison, since HqT provides only a single matching scheme, we restrict the uncertainty band on our results to use just matching scheme $(a)$. Right: comparison of our jet veto result with the jet veto result obtained by correcting HqT with the (relative) order $\alpha_{s}^{2}$ Higgs +2-jet contribution, as in eq. (7.1). Again our band includes just scheme $(a)$.

somewhat smaller than the uncertainties on the matched calculation, at least for the range of $p_{\mathrm{t}, \mathrm{veto}}$ values of practical interest. This contrasts with the situation for variables that receive contributions from all hadrons in the event, such as the beam thrust or transverse energy flow, which see large contributions from the underlying event (cf. the studies in ref. [26]).

\subsection{Comparisons with POWHEG and HqT}

Both ATLAS [11] and CMS [12] make use of POWHEG [69] for estimating their jet-veto efficiencies, interfaced to PYTHIA 6.4 (this is stated explicitly by ATLAS, we assume it to be the case for CMS). The ATLAS collaboration additionally reweights the events so as to ensure that the Higgs boson $q_{t}$ spectrum coincides with that of the HqT program [23]. In this section we compare our results to these various tools.

We start, figure 6 (left), by comparing the HqT NNLL+NNLO result for a veto on the Higgs-boson transverse momentum to our NLL+NNLO result for the jet veto. Part of the purpose of this comparison is to examine the relative sizes of the uncertainties and the benefit to be had from a NNLL resummation. In order for the comparison to be consistent with HqT, which has just a single matching scheme, our uncertainty band here includes only scale variation with scheme (a), but not the central values from the other schemes. At low values of $p_{\mathrm{t} \text {,veto }} \lesssim 20 \mathrm{GeV}$, it appears that there are clear benefits to be had from the NNLL resummation, with a significant reduction in the uncertainty as compared to a NLL 
result. On the other hand, for the practically relevant region, $p_{\mathrm{t} \text {,veto }} \sim 30 \mathrm{GeV}$, the bands are quite similar in size. This is perhaps not surprising since at this scale resummation only just starts to become relevant.

The modest size of the differences between a transverse momentum veto on the Higgsboson and one on the jet is to be expected given that the two observables differ only from $\mathcal{O}\left(\alpha_{s}^{2}\right)$ onwards. An interesting cross-check of the consistency between the two approaches can be obtained by taking the HqT calculation of the Higgs transverse-momentum and correcting it with the $\alpha_{s}^{2}$ difference between Higgs and jet transverse momentum distributions. Some care is needed in order not to introduce divergences at low $p_{t}$, and we use the following matching procedure

$$
\epsilon_{\text {jet }}^{(\mathrm{HqT}+\mathrm{H} 2 \mathrm{j} @ \mathrm{LO})}\left(p_{\mathrm{t}, \text { veto }}\right)=\left(1+\frac{\Sigma_{2, \mathrm{jet}}\left(p_{\mathrm{t}, \mathrm{veto}}\right)-\Sigma_{2, \mathrm{Higgs}}\left(p_{\mathrm{t}, \mathrm{veto}}\right)}{\sigma_{0}}\right) \epsilon_{\text {Higgs }}^{\mathrm{HqT})}\left(p_{\mathrm{t}, \mathrm{veto}}\right),
$$

involving the difference of $\Sigma_{2}$ terms for the jet- $p_{t}$ and Higgs- $p_{t}$ calculations normalised to $\sigma_{0}$, as well as the Higgs $p_{t}$-veto efficiency, $\epsilon_{\text {Higgs }}^{(\mathrm{HqT})}\left(p_{\text {t,veto }}\right)$. While such a matching does break the resummation accuracy, one may still expect it to have some meaning in the region of intermediate transverse momenta. The result is shown in figure 6 (right) as the band labelled "HqT matched with H2j@LO", using the MCFM calculation of H+2-jets at LO. It is in reasonable agreement with our NLL+NNLO calculation. This helps illustrate the consistency between the different tools. The study can be extended to NLO in the $\mathrm{H}+2$-jet calculation [20], and the results appear broadly similar, though care is needed in the treatment of technical infrared cutoffs in the $\mathrm{H}+2$-jet calculation.

Let us now turn to POwHEG. ${ }^{1}$ Here we use default renormalisation and factorisation scales equal to $M_{H} / 2$, as in our fixed order and resummed calculations. Figure 7 (left) shows the results, showered with 3 different commonly used tunes of PYTHIA, Perugia 2011, Z2 [70] and AMBT1 [71]. For the $p_{t}$ values of interest, all three are contained within the NLL+NNLO uncertainty band, though systematically above its central value.

To probe the uncertainties in POWHEG plus PYTHIA, we followed the suggestion in refs. [10, 72], and varied renormalisation and factorisation scale independently around $M_{H} / 2$ as described in eq.(3.14) and fixed the parameter hfact to $h=M_{H} / 1.2$. With these choices the Higgs transverse momentum spectrum of POWHEG+PYTHIA yields a reasonable agreement in shape with that of HqT. We recall that in POWHEG it is possible to split the real radiation contribution $R$ into a shower part $R^{s}$ and a finite one $R^{f}$, with the requirement that $R^{s} \rightarrow R$ at small transverse momenta. Choosing a finite hfact (rather than hfact $=\infty)$ means that $R^{s}=h^{2} /\left(h^{2}+p_{T, H}^{2}\right) R$ and $R^{f}=p_{T, H}^{2} /\left(h^{2}+p_{T, H}^{2}\right) R$. Therefore this choice mainly affects the large transverse momentum region. Since the transverse momentum spectrum of shower events is not affected by scale variations, while that of finite events is, this splitting provides one way of assessing of the uncertainties in POWHEG.

In figure 7 (left) this is illustrated as a band for the case where POWHEG is interfaced to PYTHIA with the Perugia 2011 tune. This band is significantly narrower than our uncertainty band and one also observes that for low values of $p_{\mathrm{t} \text {,veto, }}$, it does not quite

\footnotetext{
${ }^{1}$ We used revision 1683 .
} 

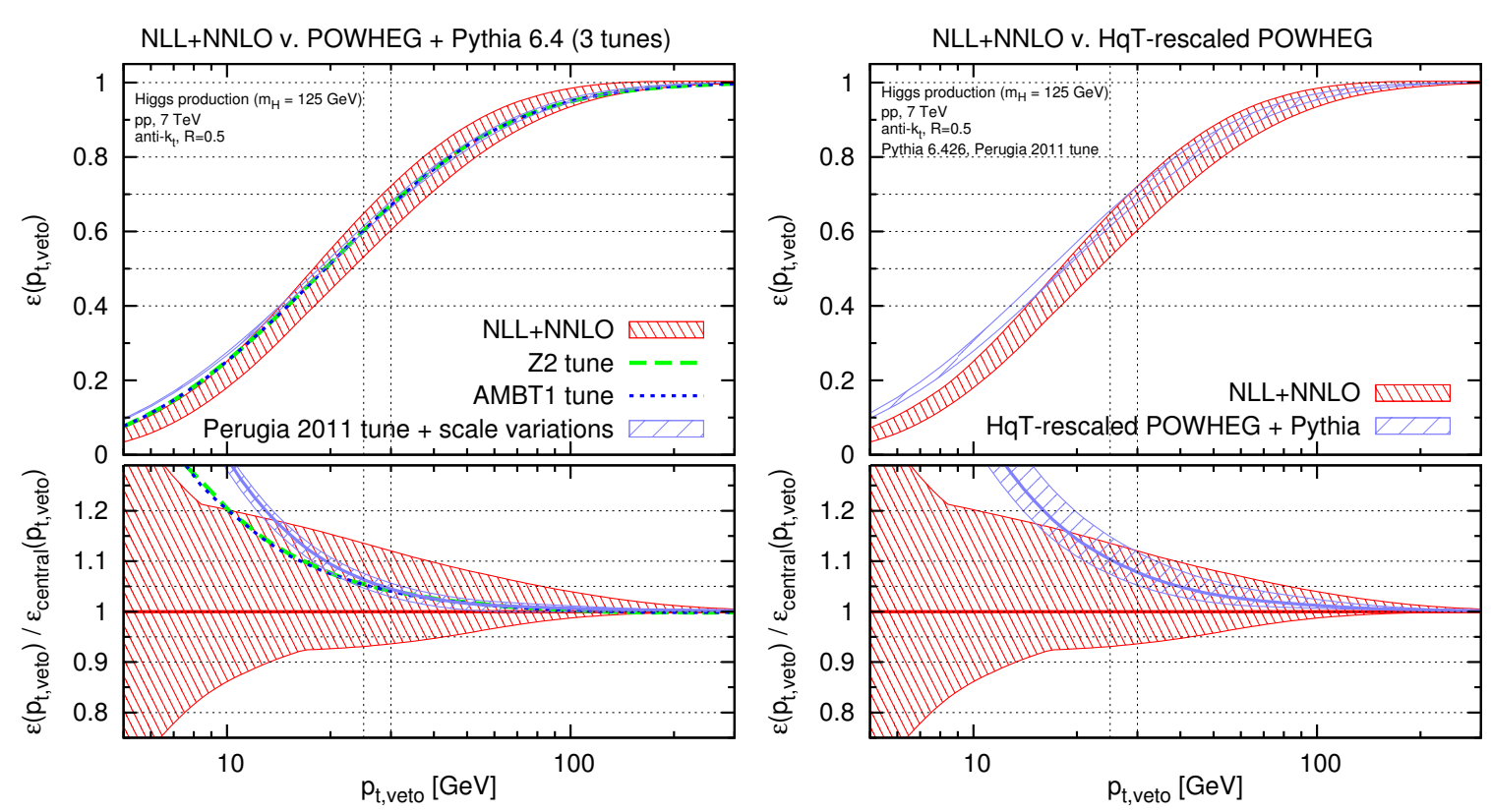

Figure 7. Left: comparison of POWHEG + PYTHIA 6.426 with our NLL+NNLO predictions; results are shown for three Pythia tunes. One of them, Perugia 2011, is displayed as a band, formed by the envelope of renormalisation and factorisation scale variations (eq. (3.14)). Right: comparison of the HqT-rescaled POWHEG + PYTHIA combination (Perugia 2011 tune) with our NLL+NNLO results. POWHEG and HqT are always used with MSTW2008NNLO PDFs.

encompass the different tunes. We tend to believe that the POWHEG uncertainty band represents an underestimate of the true uncertainties. This suggests that it might be valuable to investigate other sources of uncertainty, for example variations of hfact.

The right-hand plot of figure 7 shows the impact of reweighting events so as to ensure that the final Higgs $p_{t}$ distribution agrees with the output of the NNLL+NNLO HqT program. In this procedure, every (originally unweighted) event $i$ from POWHEG+PYTHIA is assigned a weight $w_{i}$

$$
w_{i}=\left(\left.\frac{d \sigma^{\mathrm{HqT}}}{d p_{t, H}}\right|_{p_{t, H}^{(i)}}\right) /\left(\left.\frac{d \sigma^{\text {POWHEG+Pythia }}}{d p_{t, H}}\right|_{p_{t, H}^{(i)}}\right)
$$

where $p_{t, H}^{(i)}$ is the transverse momentum of the Higgs boson in event $i$. The result of the weighting for the main tune we consider, Perugia 2011, is to increase the efficiency a little, bringing it close the upper edge of our (full) band. The reweighted POWHEG+PYTHIA band is broader than that before reweighting, but still smaller than ours. This is natural given that HqT considers just a single matching scheme as compared to our multiple schemes. Note also that the reweighting of POWHEG + PYTHIA with HqT breaks the NNLO accuracy of the HqT calculation, unlike the procedure of eq. (7.1).

Finally, we remark that while here we have considered just the POWHEG+PYTHIA results for NLO-matched parton showers, comparisons with MC@NLO [31] showered with HERWIG [73] are to be found in ref. [10]. 


\section{Conclusions}

In this article we have examined resummations and matching to fixed order calculations for jet vetoes in Higgs-Boson and Z-boson production. The NLL resummation has a particularly simple form, reducing to just a Sudakov form factor. Such a simplification had been observed in the past for the two-jet rate with the Cambridge algorithm in $e^{+} e^{-}$ collisions. Here the result holds for a broad class of jet algorithms, independently of the jet radius.

To make phenomenological use of our result it was necessary to match the resummation with a fixed-order calculation, especially because experimentally relevant jet-veto scales are at the edge of the region where resummation starts to become relevant. One may express the result either as a jet-vetoed cross section or a jet-veto efficiency. We chose to concentrate on the latter for two reasons: firstly, the efficiency is closely related to the concept behind a Sudakov resummation; secondly, the perturbative structure of the efficiency provides useful handles when estimating uncertainties. The uncertainties on the efficiency should, we believe, be essentially uncorrelated from those on the total cross section, making it straightforward to also derive uncertainties on vetoed cross sections.

For $Z$-boson production, the jet-veto efficiency turns out to be rather stable. For Higgs production, however, even if the NLL+NNLO matching provides some degree of stabilisation of the results relative to just NNLO, the final uncertainties are not negligible, cf. table 3. The problem can be traced back to ambiguities in how one formulates the fixed-order result for the efficiency and is closely connected with the poor convergence of the perturbative series for the total cross section. Essentially, considering that the known NNLO corrections to the total Higgs cross section are about 20\%, one can expect that the unknown NNLO corrections to the 1-jet rate can also easily be of order $20 \%$ (and distinct from the uncertainty on the total cross section, associated with unknown NNNLO terms), which then inevitably propagates into the jet-veto efficiency. This issue is generally not addressed in existing calculations, with the exception of ref. [57], which approaches it from a somewhat different point of view. From the perspective of resummations, such effects are $\mathrm{N}^{3} \mathrm{LL}$, and so appear to be beyond current technology. Steps towards NNLL accuracy, specifically terms related to the choice of jet definition, are discussed in the appendix.

Given the NLL+NNLO calculation, there were various phenomenological questions that could be addressed. On one hand, it was useful to establish how directly the result could be applied to data. Effects of potential relevance include finite detector acceptance and non-perturbative corrections. Based on Monte Carlo simulations, both appear to be modest compared to other remaining uncertainties. We also compared our results to the predictions from tools used by the LHC experiments, such as the NNLL+NNLO Higgs-boson $p_{t}$ resummations from the HqT program and the NLO parton-shower generator POWHEG (with PYTHIA), with or without rescaling corrections from the HqT program. We observe a pattern of slightly higher efficiencies with these tools than in our NLL+NNLO calculation, but all remain consistent to within uncertainties. 


\section{Acknowledgments}

We thank Simone Alioli, Babis Anastasiou, Emanuele Di Marco, Günther Dissertori, Massimiliano Grazzini, Pier Fracesco Monni, Paolo Nason, Vivek Sharma and Maiko Takahashi for fruitful discussions and Matthias Neubert and Thomas Becher for prompting us to complete this work in a timely fashion and for comments on the manuscript. We are grateful to CERN (AB and GZ), the GGI Institute (AB and GZ) and ETH Zürich (GPS and GZ) for hospitality while part of this work was carried out. This work was initiated while AB was at the ETH Zürich. GZ is supported by the British Science and Technology Facilities Council. The work of GPS is supported in part by the Agence Nationale de la Recherche under contract ANR-09-BLAN-0060. Both acknowledge the support also of grant PITNGA-2010-264564 from the European Commission.

\section{A NNLL $R$-dependent terms}

The CAESAR paper [34] was primarily concerned with NLL resummation. Nevertheless, it did provide certain formulae that are of use in a NNLL context, as part of the verification of the particular classes of terms that could be neglected in a NLL calculation.

It is our understanding that these formulae provide key pieces that are missing in order to relate existing NNLL resummations for the Higgs or vector boson $p_{t}$ distribution to a NNLL resummation of the jet veto efficiency and specifically the dependence of the NNLL corrections on the jet algorithm and radius.

While it is beyond the scope of this article to provide a full NNLL analysis of the jet veto efficiency, we believe it is useful, nevertheless, to make the NNLL $R$-dependent results available.

There are two sources of correction, related to the fact that the emission of two softcollinear gluons can be separated into two parts: an independent-emission term and a correlated emission term, the latter being non-zero only when the two gluons are close in rapidity:

$$
\mathcal{M}_{g g}^{2}\left(k_{1}, k_{2}\right)=\mathcal{M}_{g}^{2}\left(k_{1}\right) \mathcal{M}_{g}^{2}\left(k_{2}\right)+\widetilde{\mathcal{M}}_{g g}^{2}\left(k_{1}, k_{2}\right),
$$

where $\mathcal{M}_{g}^{2}(k)$ is the factorised matrix element for the emission of a single gluon and $\widetilde{\mathcal{M}}_{g g}^{2}\left(k_{1}, k_{2}\right)$ is the correlated-emission part of two-gluon emission matrix element in the limit where both are soft and collinear with respect to the beam direction. There is also a corresponding correlated-emission component for the production of a quark-antiquark pair, $\widetilde{\mathcal{M}}_{q \bar{q}}^{2}$. We will use $\widetilde{\mathcal{M}}^{2}$ to denote $\widetilde{\mathcal{M}}_{g g}^{2}+2 \widetilde{\mathcal{M}}_{q \bar{q}}^{2}$. For double soft (and collinear) emission off a quark line, the non-trivial correlated part of the matrix element was given as early as refs. [74, 75] and was rederived for more general event structures in refs. [76, 77]. Apart from colour factors $\left(C_{F}^{2}, C_{F} C_{A}, C_{F} T_{R} n_{f}\right.$ respectively for independent and correlated gluon and quark-pair emission from a quark line; $C_{A}^{2}, C_{A}^{2}$ and $C_{A} T_{R} n_{f}$ for emission from a gluon line), the matrix elements are the same regardless of whether the gluon pair or $q \bar{q}$ pair are emitted from a quark line or a gluon line, as can be seen clearly in the formulae of ref. [77]. 


\section{A.1 Correlated emission}

We start with the correlated-emission component, and in particular from eq. (D.8) of the CAESAR paper, which provides the NNLL contribution associated with the presence of any number of independent emissions and one correlated pair:

$$
\begin{aligned}
\mathcal{F}^{\text {correl }}= & \exp \left(-\int_{\epsilon v}^{v}[d k] \mathcal{M}_{g, r c}^{2}(k)\right) \times \\
& \times \sum_{n=0} \frac{1}{n !}\left(\prod_{i=1}^{n} \int_{\epsilon v}\left[d k_{i}\right] \mathcal{M}_{g, r c}^{2}\left(k_{i}\right)\right) \frac{1}{2 !} \int\left[d k_{a}\right]\left[d k_{b}\right] \widetilde{\mathcal{M}}_{g g, r c}^{2}\left(k_{a}, k_{b}\right) \times \\
& \times\left[\Theta\left(v-V\left(k_{1}, \ldots, k_{n}, k_{a}, k_{b}\right)\right)-\Theta\left(v-V\left(k_{1}, \ldots, k_{n}, k_{a}+k_{b}\right)\right)\right] .
\end{aligned}
$$

This formula can be understood as the correction that arises when an observable is sensitive to the kinematics of the individual $a$ and $b$ partons rather than just their sum. For the resummation of a sufficiently inclusive quantity, notably the boson $p_{t}$ distribution, it is explicitly zero. Consequently, the calculation of this term should allow one to relate the jet-veto resummation to the boson $p_{t}$ resummation. ${ }^{2}$

For compactness we have not explicitly written the $\{\tilde{p}\}$ argument to $V(\ldots)$ in eq. (A.2). We work in a limit where $\alpha_{s} \ll 1, \ln 1 / v \gg 1$ and $\alpha_{s} \ln 1 / v$ is finite. The parameter $\epsilon$, which serves as a regularisation cutoff, is to be taken $\epsilon \ll 1$, but also such that $\alpha_{s} \ln 1 / \epsilon \ll 1$. In this limit the phase space integrals $\left[d k_{i}\right]$ essentially extend up to a rapidity $\left|y_{i}\right| \lesssim \ln 1 / v$ and the dependence of the precise upper rapidity limit on a given parton's transverse momentum will turn out to be irrelevant at our accuracy. Additionally, to within a factor $\mathcal{O}(\epsilon)$, all emissions have a $p_{t} \sim v M_{B} \equiv p_{\text {t,veto }}\left(v=p_{\text {t,veto }} / M_{B}\right.$ and $M_{B}$, we recall, is the boson mass). The matrix elements include a subscript " $r c$ " to indicate that the strong coupling is to be evaluated at a scale of order $v M_{B}$.

A NNLL contribution arises only when all emissions $i=1 \ldots n$ are well separated from each other and from $a$ and $b$. For the case of the hardest jet's $p_{t}$, we then have

$$
\begin{aligned}
\Theta\left(v-V\left(k_{1}, \ldots, k_{n}, k_{a}, k_{b}\right)\right) & =\left[\prod_{i=1}^{n} \Theta\left(v-V\left(k_{i}\right)\right)\right] \Theta\left(v-V\left(k_{a}, k_{b}\right)\right), \\
\Theta\left(v-V\left(k_{1}, \ldots, k_{n}, k_{a}+k_{b}\right)\right) & =\left[\prod_{i=1}^{n} \Theta\left(v-V\left(k_{i}\right)\right)\right] \Theta\left(v-V\left(k_{a}+k_{b}\right)\right) .
\end{aligned}
$$

Making use of the fact that

$$
\sum_{n=0} \frac{1}{n !}\left(\prod_{i=1}^{n} \int_{\epsilon v}\left[d k_{i}\right] \mathcal{M}_{g, r c}^{2}\left(k_{i}\right) \Theta\left(v-V\left(k_{i}\right)\right)\right)=\exp \left(\int_{\epsilon v}^{v}[d k] \mathcal{M}_{g, r c}^{2}(k)\right)
$$

we can then rewrite eq. (A.2) simply as

$$
\mathcal{F}^{\text {correl }}=\frac{1}{2 !} \int\left[d k_{a}\right]\left[d k_{b}\right] \widetilde{\mathcal{M}}_{g g, r c}^{2}\left(k_{a}, k_{b}\right)\left[\Theta\left(v-V\left(k_{a}, k_{b}\right)\right)-\Theta\left(v-V\left(k_{a}+k_{b}\right)\right)\right],
$$

i.e. it reduces to a pure two-gluon result (with a running-coupling).

\footnotetext{
${ }^{2}$ We will not discuss here the effects of hard collinear emission off two different legs [78], which can introduce an extra source of differences between boson and jet $p_{t}$ resummations.
} 
It is simplest to first evaluate the leading $R$-dependence of this formula in the limit of a small jet radius $R$. When the angle between the two partons $a$ and $b$ is small, $\Delta_{a b}^{2}=$ $\left(y_{a}-y_{b}\right)^{2}+\left(\phi_{a}-\phi_{b}\right)^{2} \ll 1$, we can write the phase-space and matrix element as

$$
\begin{aligned}
{\left[d k_{a}\right]\left[d k_{b}\right] \widetilde{\mathcal{M}}_{g g, r c}^{2}\left(k_{a}, k_{b}\right)=} & \frac{2 C \alpha_{s}\left(k_{t, a b}\right)}{\pi} \frac{d k_{t, a b}}{k_{t, a b}} \frac{d \phi_{a b}}{2 \pi} d y_{a b} \times \\
& \times \frac{\alpha_{s}\left(k_{t, a b} \Delta_{a b}\right)}{\pi} \frac{d \Delta_{a b}}{\Delta_{a b}} d z\left(P_{g g}(z)+2 n_{f} P_{q g}(z)\right),
\end{aligned}
$$

where $C=C_{F}$ or $C_{A}$ depending on the nature of the beam partons, $k_{a b} \equiv k_{a}+k_{b}$ and the $P_{g g}$ and $P_{q g}$ are the real parts of the usual leading order splitting functions:

$$
\begin{aligned}
& P_{g g}(z)=2 C_{A}\left(\frac{z}{1-z}+\frac{1-z}{z}+z(1-z)\right), \\
& P_{q g}(z)=T_{R}\left(z^{2}+(1-z)^{2}\right) .
\end{aligned}
$$

With these variables, for $\Delta_{a b} \ll 1$, the difference of $\Theta$ functions in eq. (A.5) reduces to

$$
\begin{aligned}
& {\left[\Theta\left(v-V\left(k_{a}, k_{b}\right)\right)-\Theta\left(v-V\left(k_{a}+k_{b}\right)\right)\right]=} \\
& \Theta\left(\Delta_{a b}-R\right)\left[\Theta\left(v M_{B}-\max (z, 1-z) k_{t, a b}\right)-\Theta\left(v M_{B}-k_{t, a b}\right)\right],
\end{aligned}
$$

as long as one restricts one's attention to jet algorithms from the generalised- $k_{t}$ family. We can now rewrite eq. (A.5) as

$$
\begin{aligned}
\mathcal{F}^{\mathrm{correl}}=\frac{1}{2 !} \int & \left(\frac{2 C \alpha_{s}\left(k_{t, a b}\right)}{\pi} \frac{d \phi_{a b}}{2 \pi} d y_{a b}\right)\left(\frac{d \Delta_{a b}}{\Delta_{a b}} \Theta\left(\Delta_{a b}-R\right)\right) \times \\
& \times \frac{\alpha_{s}\left(k_{t, a b} \Delta_{a b}\right)}{\pi} d z\left(P_{g g}(z)+2 n_{f} P_{q g}(z)\right) \times \\
& \times\left(\frac{d k_{t, a b}}{k_{t, a b}}\left[\Theta\left(v M_{B}-\max (z, 1-z) k_{t, a b}\right)-\Theta\left(v M_{B}-k_{t, a b}\right)\right]\right) .
\end{aligned}
$$

A first point is that $k_{t, a b}$ will be limited to be of order $v M_{B}=p_{\text {t,veto }}$. Secondly, while we take $R<\Delta_{a b} \ll 1$, we will still assume that $\alpha_{s} \ln R$ is negligible. Accordingly we can replace each of the running couplings with $\alpha_{s}\left(p_{\mathrm{t}, \mathrm{veto}}\right)$. This puts us in a position to carry out the integrations in each of the three lines of eq. (A.9) independently. The contents of the first round brackets on the first line give $R_{B}^{\prime}=4 C \alpha_{s}\left(p_{\text {t,veto }}\right) / \pi \ln 1 / v$; the second set of round brackets on that line gives $-\ln R$ (for now we neglect the $\mathcal{O}(1)$ contribution from the ill-defined upper limit in $\left.\Delta_{a b}\right)$; the last line gives $\ln (1 / \max (z, 1-z))$. We are therefore left with

$$
\mathcal{F}^{\text {correl }}=R_{B}^{\prime}\left(\ln \frac{1}{R}+\mathcal{O}(1)\right) \frac{\alpha_{s}\left(p_{\text {t,veto }}\right)}{\pi} \int_{0}^{1} d z \frac{1}{2 !}\left(P_{g g}(z)+2 n_{f} P_{q g}(z)\right) \ln \frac{1}{\max (z, 1-z)} .
$$

This is straightforward to evaluate and gives

$$
\begin{aligned}
\mathcal{F}^{\text {correl }} & =R_{B}^{\prime} \frac{\alpha_{s}\left(p_{\mathrm{t}, \mathrm{veto}}\right)}{\pi}\left(C_{A} \frac{12 \pi^{2}+132 \ln 2-131}{72}+n_{f} T_{R} \frac{23-24 \ln 2}{36}\right)\left(\ln \frac{1}{R}+\mathcal{O}(1)\right), \\
& \simeq R_{B}^{\prime} \frac{\alpha_{s}\left(p_{\mathrm{t}, \mathrm{veto}}\right)}{\pi}\left(1.09626 C_{A}+0.176791 n_{f} T_{R}\right)\left(\ln \frac{1}{R}+\mathcal{O}(1)\right) .
\end{aligned}
$$


For a complete evaluation of the $\mathcal{F}^{\text {correl }}$, we start again from eq. (A.5). We multiply and divide $\widetilde{\mathcal{M}}_{g g, r c}^{2}\left(k_{a}, k_{b}\right)$ by a factor $\mathcal{M}_{g, r c}^{2}\left(k_{a}\right) \mathcal{M}_{g, r c}^{2}\left(k_{b}\right)$, and make use of

$$
[d k] \mathcal{M}_{g, r c}^{2}(k)=\frac{2 \alpha_{s}\left(k_{t}\right) C}{\pi} \frac{d k_{t}}{k_{t}} \frac{d \phi}{2 \pi} d y
$$

We then replace the integration measure $d k_{t, b} / k_{t, b} d \phi_{b} d y_{b}$ with $d \zeta / \zeta d \Delta \phi d \Delta y$ where $\zeta=$ $k_{t, b} / k_{t, a}, \Delta \phi=\phi_{b}-\phi_{a}, \Delta y=y_{b}-y_{a}$. This gives us

$$
\begin{aligned}
\mathcal{F}^{\mathrm{correl}}=\frac{1}{2 !} \int & \left(\frac{2 \alpha_{s} C}{\pi} \frac{d \phi_{a}}{2 \pi} d y_{a}\right) \frac{2 \alpha_{s} C}{\pi} \int_{0}^{\infty} \frac{d \zeta}{\zeta} \int_{-\pi}^{\pi} \frac{d \Delta \phi}{2 \pi} \int_{-\infty}^{\infty} d \Delta y \times \\
& \times \frac{\widetilde{\mathcal{M}}_{g g}^{2}\left(k_{a}, k_{b}\right)}{\mathcal{M}_{g}^{2}\left(k_{a}\right) \mathcal{M}_{g}^{2}\left(k_{b}\right)} \frac{d k_{t, a}}{k_{t, a}}\left[\Theta\left(v-V\left(k_{a}, k_{b}\right)\right)-\Theta\left(v-V\left(k_{a}+k_{b}\right)\right)\right] .
\end{aligned}
$$

As before, the first factor in round brackets on the first line will integrate to give $R_{B}^{\prime}$. The difference of $\Theta$ functions on the second line will be non-zero only when the two partons are separated by $\Delta \phi^{2}+\Delta y^{2}>R^{2}$ (this statement holds only for a recombination scheme such as the $E$-scheme, which directly sums 4 -vectors). In the ratio of matrix elements we drop the " $r c$ " subscript, since at our accuracy running coupling effects are entirely accounted for in the scale choice that we will make, $\alpha_{s}\left(p_{\mathrm{t}, \mathrm{veto}}\right)$, for the explicit factors of $\alpha_{s}$ (cf. the discussion in the small- $R$ limit). Observing that $k_{t, a b}^{2} / k_{t, a}^{2}$ is independent of $k_{t, a}^{2}$ and equal to $\left(1+\zeta^{2}+2 \zeta \cos \Delta \phi\right)$, we can then perform the $k_{t, a}$ integration together with the $\Theta$-function constraints in the square brackets to yield a factor

$$
\frac{1}{2} \ln \left[\frac{1+\zeta^{2}+2 \zeta \cos \Delta \phi}{\max \left\{\zeta^{2}, 1\right\}}\right]
$$

Our result for $\mathcal{F}^{\text {correl }}$ is then

$$
\begin{aligned}
\mathcal{F}^{\text {correl }}=R_{B}^{\prime} \frac{2 \alpha_{s}\left(p_{\mathrm{t}, \mathrm{veto}}\right) C}{\pi} \int_{0}^{\infty} & \frac{d \zeta}{\zeta} \int_{-\pi}^{\pi} \frac{d \Delta \phi}{2 \pi} \int_{-\infty}^{\infty} d \Delta y \Theta\left(\Delta \phi^{2}+\Delta y^{2}-R^{2}\right) \times \\
& \times \frac{1}{2 !} \frac{\widetilde{\mathcal{M}}_{g g}^{2}\left(k_{a}, k_{b}\right)}{\mathcal{M}_{g}^{2}\left(k_{a}\right) \mathcal{M}_{g}^{2}\left(k_{b}\right)} \frac{1}{2} \ln \left[\frac{1+\zeta^{2}+2 \zeta \cos \Delta \phi}{\max \left\{\zeta^{2}, 1\right\}}\right] .
\end{aligned}
$$

In both $R_{B}^{\prime}$ and the explicit factor of $\alpha_{s}$, the coupling is evaluated at scale $p_{\mathrm{t} \text {,veto }}$, as before. The derivative with respect to $R$ of eq. (A.15) can be straightforwardly evaluated as an expansion in powers of $R$. Integrating that expansion gives us a result for $\mathcal{F}^{\text {correl }}$ that is missing a constant of integration. This constant can easily be determined through a numerical integration of eq. (A.15), which also allows for a check of the range of validity 

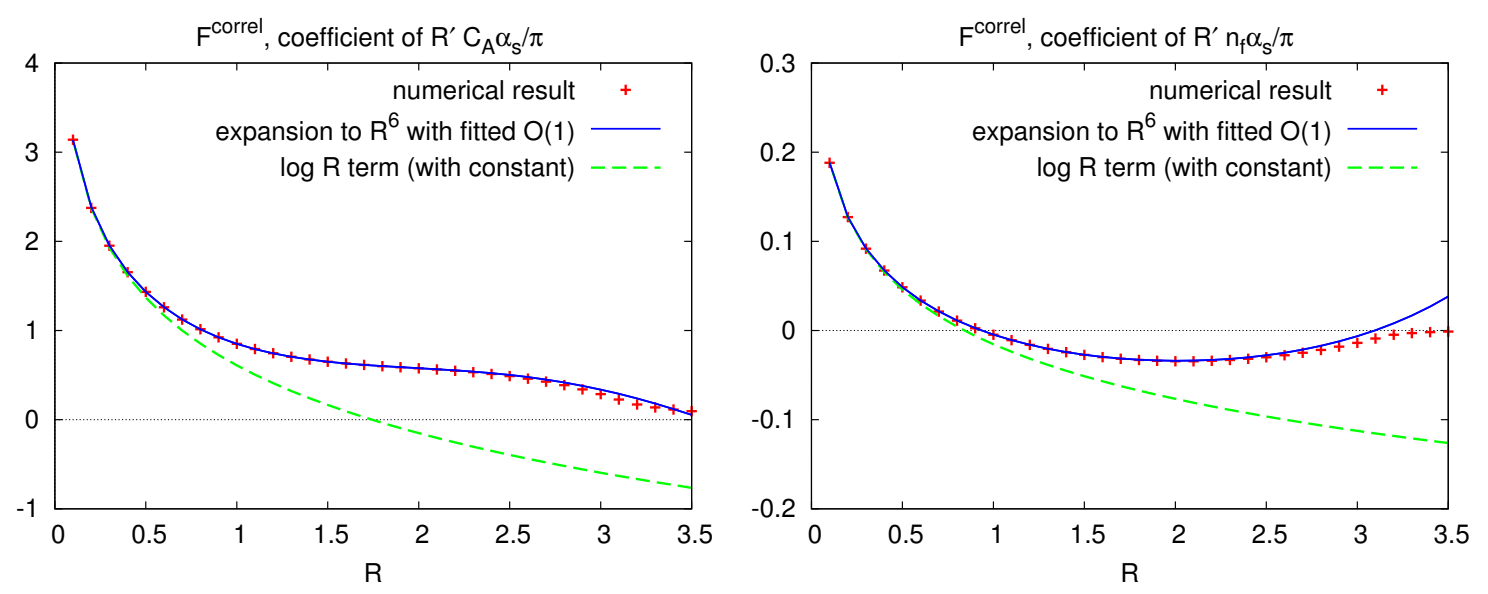

Figure 8. Comparison of the numerical and small- $R$ analytical determinations of the $C_{A}$ and $C_{F}$ pieces of $\mathcal{F}^{\text {correl }}$.

of the power series in $R$. The result that we obtain is

$$
\begin{aligned}
\mathcal{F}^{\text {correl }}= & R_{B}^{\prime} \frac{\alpha_{s}\left(p_{\text {t,veto }}\right)}{\pi}\left[\frac{\left(-131+12 \pi^{2}+132 \ln 2\right) C_{A}}{72} \ln \frac{1.74}{R}+\frac{(23-24 \ln 2) n_{f}}{72} \ln \frac{0.84}{R}\right. \\
& +\frac{\left(1429+3600 \pi^{2}+12480 \ln 2\right) C_{A}+(3071-1680 \ln 2) n_{f}}{172800} R^{2} \\
& +\frac{\left(-9383279-117600 \pi^{2}+1972320 \ln 2\right) C_{A}+2(178080 \ln 2-168401) n_{f}}{406425600} R^{4} \\
& \left.+\frac{(74801417-33384960 \ln 2) C_{A}+(7001023-5322240 \ln 2) n_{f}}{97542144000} R^{6}+O\left(R^{8}\right)\right] .
\end{aligned}
$$

This result is compared to the full numerical determination in figure 8 .

\section{A.2 Independent emission}

In the case of the jet observables that we have been discussing, the starting point for the independent-emission correction is

$$
\begin{aligned}
\mathcal{F}^{\text {indep }}= & \exp \left(-\int_{\epsilon v}^{v}[d k] \mathcal{M}_{g, r c}^{2}(k)\right) \times \\
& \times \sum_{n=0} \frac{1}{n !}\left(\prod_{i=1}^{n} \int_{\epsilon v}\left[d k_{i}\right] \mathcal{M}_{g, r c}^{2}\left(k_{i}\right) \Theta\left(v-V\left(k_{i}\right)\right)\right) \frac{1}{2 !} \int\left[d k_{a}\right]\left[d k_{b}\right] \mathcal{M}_{g, r c}^{2}\left(k_{a}\right) \mathcal{M}_{g, r c}^{2}\left(k_{b}\right) \times \\
& \times\left[\Theta\left(v-V\left(k_{a}, k_{b}\right)\right)-\Theta\left(v-V\left(k_{a}\right)\right) \Theta\left(v-V\left(k_{b}\right)\right)\right] .
\end{aligned}
$$

The second term in square brackets on the last line corresponds to the approximation made in obtaining $\mathcal{F}=1$ at NLL; the first term corresponds to the actual value of the observable. The evaluation of this formula largely follows the working given above in the correlated emission case, with the main difference that now the only region that contributes is that 
where the two gluons have $\Delta_{a b}<R$. The result for $R<\pi$ is

$$
\begin{aligned}
\mathcal{F}^{\text {indep }} & =-R_{B}^{\prime} \frac{\alpha_{s}\left(p_{\mathrm{t}, \text { veto }}\right) C}{\pi} \frac{1}{2 !} \int_{0}^{R} \Delta_{a b} d \Delta_{a b} \int_{0}^{2 \pi} \frac{d \psi}{2 \pi} \int_{0}^{\infty} \frac{d \zeta}{\zeta} \ln \left[\frac{1+\zeta^{2}+2 \zeta \cos \left(\Delta_{a b} \cos \psi\right)}{\max \left\{\zeta^{2}, 1\right\}}\right] \\
& \left.=R_{B}^{\prime} \frac{\alpha_{s}\left(p_{\mathrm{t}, \text { veto }}\right) C}{\pi}\left(-\frac{\pi^{2} R^{2}}{12}+\frac{R^{4}}{16}\right) .18 \mathrm{a}\right)
\end{aligned}
$$

Open Access. This article is distributed under the terms of the Creative Commons Attribution License which permits any use, distribution and reproduction in any medium, provided the original author(s) and source are credited.

\section{References}

[1] ATLAS collaboration, G. Aad et al., Combined search for the standard model Higgs boson using up to $4.9 \mathrm{fb}^{-1}$ of pp collision data at $\sqrt{s}=7 \mathrm{TeV}$ with the ATLAS detector at the LHC, Phys. Lett. B 710 (2012) 49 [arXiv:1202.1408] [INSPIRE].

[2] CMS collaboration, S. Chatrchyan et al., Combined results of searches for the standard model Higgs boson in pp collisions at $\sqrt{s}=7$ TeV, Phys. Lett. B 710 (2012) 26 [arXiv: 1202.1488] [INSPIRE].

[3] Tevnph (Tevatron New Phenomena and Higgs Working Group), CDF and D0 collaborations, Combined CDF and D0 search for standard model Higgs boson production with up to $10.0 \mathrm{fb}^{-1}$ of data, arXiv:1203.3774 [INSPIRE].

[4] P.W. Higgs, Broken symmetries, massless particles and gauge fields, Phys. Lett. 12 (1964) 132 [InSPIRE].

[5] P.W. Higgs, Broken symmetries and the masses of gauge bosons, Phys. Rev. Lett. 13 (1964) 508 [inSPIRE].

[6] P.W. Higgs, Spontaneous symmetry breakdown without massless bosons, Phys. Rev. 145 (1966) 1156 [INSPIRE].

[7] F. Englert and R. Brout, Broken symmetry and the mass of gauge vector mesons, Phys. Rev. Lett. 13 (1964) 321 [INSPIRE].

[8] G.S. Guralnik, C.R. Hagen and T.W.B. Kibble, Global conservation laws and massless particles, Phys. Rev. Lett. 13 (1964) 585 [InSPIRE].

[9] LHC Higgs Cross Section Working Group collaboration, S. Dittmaier et al., Handbook of LHC Higgs cross sections: 1. Inclusive observables, arXiv:1101.0593 [INSPIRE].

[10] S. Dittmaier et al., Handbook of LHC Higgs cross sections: 2. Differential distributions, arXiv:1201.3084 [INSPIRE].

[11] ATLAS collaboration, G. Aad et al., Search for the Higgs boson in the $H \rightarrow W W^{(*)} \rightarrow \ell^{+} \nu \ell^{-} \bar{\nu}$ decay channel in pp collisions at $\sqrt{s}=7 \mathrm{TeV}$ with the ATLAS detector, Phys. Rev. Lett. 108 (2012) 111802 [arXiv:1112.2577] [INSPIRE].

[12] CMS collaboration, S. Chatrchyan et al., Search for the standard model Higgs boson decaying to a $W$ pair in the fully leptonic final state in pp collisions at $\sqrt{s}=7 \mathrm{TeV}$, Phys. Lett. B 710 (2012) 91 [arXiv:1202.1489] [InSPIRE]. 
[13] C. Anastasiou, K. Melnikov and F. Petriello, Fully differential Higgs boson production and the di-photon signal through next-to-next-to-leading order, Nucl. Phys. B 724 (2005) 197 [hep-ph/0501130] [INSPIRE].

[14] S. Catani and M. Grazzini, An NNLO subtraction formalism in hadron collisions and its application to Higgs boson production at the LHC, Phys. Rev. Lett. 98 (2007) 222002 [hep-ph/0703012] [INSPIRE].

[15] M. Grazzini, NNLO predictions for the Higgs boson signal in the $H \rightarrow W W \rightarrow l \nu l \nu$ and $H \rightarrow Z Z \rightarrow 4 l$ decay channels, JHEP 02 (2008) 043 [arXiv: 0801.3232] [INSPIRE].

[16] D. de Florian, M. Grazzini and Z. Kunszt, Higgs production with large transverse momentum in hadronic collisions at next-to-leading order, Phys. Rev. Lett. 82 (1999) 5209 [hep-ph/9902483] [INSPIRE].

[17] V. Ravindran, J. Smith and W.L. Van Neerven, Next-to-leading order QCD corrections to differential distributions of Higgs boson production in hadron hadron collisions, Nucl. Phys. B 634 (2002) 247 [hep-ph/0201114] [INSPIRE].

[18] C.J. Glosser and C.R. Schmidt, Next-to-leading corrections to the Higgs boson transverse momentum spectrum in gluon fusion, JHEP 12 (2002) 016 [hep-ph/0209248] [INSPIRE].

[19] J.M. Campbell, R.K. Ellis and G. Zanderighi, Next-to-leading order Higgs + 2 jet production via gluon fusion, JHEP 10 (2006) 028 [hep-ph/0608194] [INSPIRE].

[20] J.M. Campbell, R.K. Ellis and C. Williams, Hadronic production of a Higgs boson and two jets at next-to-leading order, Phys. Rev. D 81 (2010) 074023 [arXiv:1001.4495] [INSPIRE].

[21] I.W. Stewart, F.J. Tackmann and W.J. Waalewijn, Factorization at the LHC: from PDFs to initial state jets, Phys. Rev. D 81 (2010) 094035 [arXiv:0910.0467] [INSPIRE].

[22] G. Bozzi, S. Catani, D. de Florian and M. Grazzini, The $q_{T}$ spectrum of the Higgs boson at the LHC in QCD perturbation theory, Phys. Lett. B 564 (2003) 65 [hep-ph/0302104] [INSPIRE].

[23] G. Bozzi, S. Catani, D. de Florian and M. Grazzini, Transverse-momentum resummation and the spectrum of the Higgs boson at the LHC, Nucl. Phys. B 737 (2006) 73 [hep-ph/0508068] [INSPIRE].

[24] C.F. Berger, C. Marcantonini, I.W. Stewart, F.J. Tackmann and W.J. Waalewijn, Higgs production with a central jet veto at NNLL+NNLO, JHEP 04 (2011) 092 [arXiv:1012.4480] [INSPIRE].

[25] T. Becher and M. Neubert, Drell-Yan production at small $q_{T}$, transverse parton distributions and the collinear anomaly, Eur. Phys. J. C 71 (2011) 1665 [arXiv:1007.4005] [INSPIRE].

[26] A. Papaefstathiou, J.M. Smillie and B.R. Webber, Resummation of transverse energy in vector boson and Higgs boson production at hadron colliders, JHEP 04 (2010) 084 [arXiv: 1002 .4375] [INSPIRE].

[27] S. Catani, Y.L. Dokshitzer, M. Olsson, G. Turnock and B.R. Webber, New clustering algorithm for multi-jet cross-sections in $e^{+} e^{-}$annihilation, Phys. Lett. B 269 (1991) 432 [INSPIRE].

[28] S. Catani, Y.L. Dokshitzer, M.H. Seymour and B.R. Webber, Longitudinally invariant $k_{t}$ clustering algorithms for hadron hadron collisions, Nucl. Phys. B 406 (1993) 187 [InSPIRE]. 
[29] A. Banfi, G.P. Salam and G. Zanderighi, Semi-numerical resummation of event shapes, JHEP 01 (2002) 018 [hep-ph/0112156] [INSPIRE].

[30] A. Banfi, G.P. Salam and G. Zanderighi, Resummed event shapes at hadron-hadron colliders, JHEP 08 (2004) 062 [hep-ph/0407287] [INSPIRE].

[31] S. Frixione and B.R. Webber, Matching NLO QCD computations and parton shower simulations, JHEP 06 (2002) 029 [hep-ph/0204244] [INSPIRE].

[32] P. Nason, A new method for combining NLO QCD with shower Monte Carlo algorithms, JHEP 11 (2004) 040 [hep-ph/0409146] [INSPIRE].

[33] J.C. Collins, D.E. Soper and G.F. Sterman, Transverse momentum distribution in Drell-Yan pair and $W$ and $Z$ boson production, Nucl. Phys. B 250 (1985) 199 [INSPIRE].

[34] A. Banfi, G.P. Salam and G. Zanderighi, Principles of general final-state resummation and automated implementation, JHEP 03 (2005) 073 [hep-ph/0407286] [INSPIRE].

[35] M. Cacciari, G.P. Salam and G. Soyez, The anti-kt jet clustering algorithm, JHEP 04 (2008) 063 [arXiv:0802.1189] [INSPIRE].

[36] M. Dasgupta and G.P. Salam, Resummation of nonglobal QCD observables, Phys. Lett. B 512 (2001) 323 [hep-ph/0104277] [INSPIRE].

[37] M. Dasgupta and G.P. Salam, Accounting for coherence in interjet $E_{t}$ flow: a case study, JHEP 03 (2002) 017 [hep-ph/0203009] [INSPIRE].

[38] M. Dasgupta and G.P. Salam, Resummed event shape variables in DIS, JHEP 08 (2002) 032 [hep-ph/0208073] [INSPIRE].

[39] S.D. Ellis and D.E. Soper, Successive combination jet algorithm for hadron collisions, Phys. Rev. D 48 (1993) 3160 [hep-ph/9305266] [INSPIRE].

[40] Y.L. Dokshitzer, G.D. Leder, S. Moretti and B.R. Webber, Better jet clustering algorithms, JHEP 08 (1997) 001 [hep-ph/9707323] [INSPIRE].

[41] M. Wobisch and T. Wengler, Hadronization corrections to jet cross-sections in deep inelastic scattering, hep-ph/9907280 [INSPIRE].

[42] M. Wobisch, Measurement and QCD analysis of jet cross-sections in deep inelastic positron proton collisions at $\sqrt{s}=300 \mathrm{GeV}$, DESY-THESIS-2000-049 [INSPIRE].

[43] S. Catani, B.R. Webber and G. Marchesini, QCD coherent branching and semiinclusive processes at large $x$, Nucl. Phys. B 349 (1991) 635 [INSPIRE].

[44] G.P. Salam and G. Soyez, A practical seedless infrared-safe cone jet algorithm, JHEP 05 (2007) 086 [arXiv:0704.0292] [INSPIRE].

[45] S. Catani, L. Trentadue, G. Turnock and B.R. Webber, Resummation of large logarithms in $e^{+} e^{-}$event shape distributions, Nucl. Phys. B 407 (1993) 3 [InSPIRE].

[46] M. Dasgupta and G.P. Salam, Resummed event shape variables in DIS, JHEP 08 (2002) 032 [hep-ph/0208073] [INSPIRE].

[47] A.D. Martin, W.J. Stirling, R.S. Thorne and G. Watt, Parton distributions for the LHC, Eur. Phys. J. C 63 (2009) 189 [arXiv:0901.0002] [inSPIRE].

[48] R. Gavin, Y. Li, F. Petriello and S. Quackenbush, FEWZ 2.0: a code for hadronic Z production at next-to-next-to-leading order, Comput. Phys. Commun. 182 (2011) 2388 [arXiv: 1011.3540] [INSPIRE]. 
[49] S. Catani, L. Cieri, G. Ferrera, D. de Florian and M. Grazzini, Vector boson production at hadron colliders: a fully exclusive QCD calculation at NNLO,

Phys. Rev. Lett. 103 (2009) 082001 [arXiv:0903.2120] [INSPIRE].

[50] R.V. Harlander and W.B. Kilgore, Next-to-next-to-leading order Higgs production at hadron colliders, Phys. Rev. Lett. 88 (2002) 201801 [hep-ph/0201206] [INSPIRE].

[51] C. Anastasiou and K. Melnikov, Higgs boson production at hadron colliders in NNLO QCD, Nucl. Phys. B 646 (2002) 220 [hep-ph/0207004] [INSPIRE].

[52] V. Ravindran, J. Smith and W.L. van Neerven, NNLO corrections to the total cross-section for Higgs boson production in hadron hadron collisions, Nucl. Phys. B 665 (2003) 325 [hep-ph/0302135] [inSPIRE].

[53] R. Hamberg, W.L. van Neerven and T. Matsuura, A complete calculation of the order $\alpha_{s}^{2}$ correction to the Drell-Yan K factor, Nucl. Phys. B 359 (1991) 343 [Erratum ibid. B 644 (2002) 403] [INSPIRE].

[54] J.M. Campbell and R.K. Ellis, Next-to-leading order corrections to $W+2$ jet and $Z+2$ jet production at hadron colliders, Phys. Rev. D 65 (2002) 113007 [hep-ph/0202176] [INSPIRE].

[55] S. Dawson, Radiative corrections to Higgs boson production, Nucl. Phys. B 359 (1991) 283 [INSPIRE].

[56] A. Djouadi, M. Spira and P.M. Zerwas, Production of Higgs bosons in proton colliders: QCD corrections, Phys. Lett. B 264 (1991) 440 [INSPIRE].

[57] I.W. Stewart and F.J. Tackmann, Theory uncertainties for Higgs and other searches using jet bins, Phys. Rev. D 85 (2012) 034011 [arXiv:1107.2117] [INSPIRE].

[58] S. Catani, D. de Florian, M. Grazzini and P. Nason, Soft gluon resummation for Higgs boson production at hadron colliders, JHEP 07 (2003) 028 [hep-ph/0306211] [INSPIRE].

[59] N. Kidonakis, Collinear and soft gluon corrections to Higgs production at NNNLO, Phys. Rev. D 77 (2008) 053008 [arXiv:0711.0142] [INSPIRE].

[60] V. Ahrens, T. Becher, M. Neubert and L.L. Yang, Origin of the large perturbative corrections to Higgs production at hadron colliders, Phys. Rev. D 79 (2009) 033013 [arXiv:0808.3008] [INSPIRE].

[61] V. Ahrens, T. Becher, M. Neubert and L.L. Yang, Renormalization-group improved prediction for Higgs production at hadron colliders, Eur. Phys. J. C 62 (2009) 333 [arXiv: 0809.4283] [INSPIRE].

[62] J. Baglio and A. Djouadi, Higgs production at the LHC, JHEP 03 (2011) 055 [arXiv: 1012.0530] [INSPIRE].

[63] R.W.L. Jones, M. Ford, G.P. Salam, H. Stenzel and D. Wicke, Theoretical uncertainties on $\alpha_{s}$ from event shape variables in $e^{+} e^{-}$annihilations, JHEP 12 (2003) 007 [hep-ph/0312016] [INSPIRE].

[64] B. Nobalma et al., in The SM and NLO multileg and SM MC working groups: summary report, work presented at the workshop on Physics at TeV Collider, Les Houches France, 31 May-8 June 2011.

[65] T. Sjöstrand, S. Mrenna and P.Z. Skands, PYTHIA 6.4 physics and manual, JHEP 05 (2006) 026 [hep-ph/0603175] [inSPIRE]. 
[66] P.Z. Skands, Tuning Monte Carlo generators: the Perugia tunes, Phys. Rev. D 82 (2010) 074018 [arXiv: 1005.3457] [InSPIRE].

[67] M. Cacciari and G.P. Salam, Dispelling the $N^{3}$ myth for the $k_{t}$ jet-finder, Phys. Lett. B 641 (2006) 57 [hep-ph/0512210] [INSPIRE].

[68] M. Cacciari, G.P. Salam and G. Soyez, FastJet user manual, Eur. Phys. J. C 72 (2012) 1896 [arXiv: 1111.6097] [INSPIRE].

[69] S. Alioli, P. Nason, C. Oleari and E. Re, NLO Higgs boson production via gluon fusion matched with shower in POWHEG, JHEP 04 (2009) 002 [arXiv:0812.0578] [INSPIRE].

[70] R. Field, Early LHC underlying event data - findings and surprises, arXiv:1010.3558 [INSPIRE].

[71] ATLAS collaboration, G. Aad et al., Charged-particle multiplicities in pp interactions measured with the ATLAS detector at the LHC, New J. Phys. 13 (2011) 053033 [arXiv: 1012.5104] [INSPIRE].

[72] J.M. Campbell et al., NLO Higgs boson production plus one and two jets using the POWHEG BOX, MadGraph4 and MCFM, arXiv:1202.5475 [INSPIRE].

[73] G. Corcella et al., HERWIG 6.5 release note, hep-ph/0210213 [INSPIRE].

[74] F.A. Berends and W.T. Giele, Multiple soft gluon radiation in parton processes, Nucl. Phys. B 313 (1989) 595 [inSPIRE].

[75] Y.L. Dokshitzer, G. Marchesini and G. Oriani, Measuring color flows in hard processes: beyond leading order, Nucl. Phys. B 387 (1992) 675 [INSPIRE].

[76] J.M. Campbell and E.W.N. Glover, Double unresolved approximations to multiparton scattering amplitudes, Nucl. Phys. B 527 (1998) 264 [hep-ph/9710255] [INSPIRE].

[77] S. Catani and M. Grazzini, Infrared factorization of tree level QCD amplitudes at the next-to-next-to-leading order and beyond, Nucl. Phys. B 570 (2000) 287 [hep-ph/9908523] [INSPIRE].

[78] S. Catani and M. Grazzini, QCD transverse-momentum resummation in gluon fusion processes, Nucl. Phys. B 845 (2011) 297 [arXiv:1011.3918] [INSPIRE]. 